\title{
A KAM Theorem for Hamiltonian Partial Differential Equations with Unbounded Perturbations *
}

\author{
Jianjun Liu, Xiaoping Yuan \\ School of Mathematical Sciences and Key Lab of Math. for Nonlinear Science, \\ Fudan University, Shanghai 200433, P R China
}

September 30, 2010

\begin{abstract}
We establish an abstract infinite dimensional KAM theorem dealing with unbounded perturbation vector-field, which could be applied to a large class of Hamiltonian PDEs containing the derivative $\partial_{x}$ in the perturbation. Especially, in this range of application lie a class of derivative nonlinear Schrödinger equations with Dirichlet boundary conditions and perturbed Benjamin-Ono equation with periodic boundary conditions, so KAM tori and thus quasi-periodic solutions are obtained for them.
\end{abstract}

\section{Introduction and Main Results}

Consider a Hamiltonian partial differential equation (HPDE)

$$
\dot{w}=A w+F(w),
$$

where $A w$ is linear Hamiltonian vector-field with $d:=\operatorname{ord} A>0$ and $F(w)$ is nonlinear Hamiltonian vector-field with $\tilde{d}:=\operatorname{ord} F$ and is analytic in the neighborhood of the origin $w=0$.

When $\tilde{d}=\operatorname{ord} F \leq 0$, the vector-field $F$ is called bounded perturbation. For example, in this case lie a class of nonlinear Schrödinger equations (with $\tilde{d}=0$ )

$$
\mathbf{i} u_{t}+u_{x x}+V(x) u+|u|^{2} u=0
$$

and a class of nonlinear wave equations (with $\tilde{d}=-1$ )

$$
u_{t t}-u_{x x}+V(x) u+u^{3}=0 .
$$

For the existence of KAM tori of the PDEs with bounded perturbations has been deeply and widely investigated by many authors. In this field of study there are too many references to list here. We give just two survey papers by Kuksin [8] and Bourgain [4].

When $\tilde{d}=$ ord $F>0$, the vector-field $F$ is called unbounded perturbation. According to a wellknown example, due to Lax [11] and Klainerman [5] (See also [7]), it is reasonable to assume

$$
\tilde{d} \leq d-1
$$

in order to guarantee the existence of KAM tori for the PDE. The quantity $d-\tilde{d}$ measures the strength of nonlinearity of the PDE. The smaller the $d-\tilde{d}$ is, the stronger the nonlinearity is. When $d-\tilde{d}=1$, the nonlinearity of the PDE is the strongest.

\footnotetext{
*Supported by NNSFC and 973 Program (No. 2010CB327900) and the Research Foundation for Doctor Programme.
} 
For the PDE with unbounded Hamiltonian perturbation, the only previous KAM theorem is due to Kuksin [7] where it is assumed that $d-\tilde{d}>1$. Kuksin's theorem is in [7] used to prove the persistence of the finite-gap solutions of $\mathrm{KdV}$ equation, as well as its hierarchy, subject to periodic boundary conditions. See also Kappeler-Pöschel [10]. Another KAM theorem with unbounded linear Hamiltonian perturbation is due to Bambusi-Graffi [2] where the spectrum property is investigated for the time dependent linear Schrödinger equation

$$
\mathbf{i} \dot{y}(t)=(A+\varepsilon F(t)) y(t)
$$

with $d-\tilde{d}>1$ also.

The assumption $d-\tilde{d}>1$ excludes a large class of interesting partial differential equations such as a class of derivative nonlinear Schrödinger (DNLS) equations

$$
\mathbf{i} u_{t}+u_{x x}-M_{\sigma} u+\mathbf{i} f(u, \bar{u}) u_{x}=0
$$

subject to Dirichlet boundary conditions, and perturbed Benjamin-Ono (BO) equation

$$
u_{t}+\mathscr{H} u_{x x}-u u_{x}+\text { perturbation }=0
$$

subject to periodic boundary conditions, where $\mathscr{H}$ is the Hilbert transform. In both cases, $d-\tilde{d}=$ 1. In the present paper, we will construct a KAM theorem including $d-\tilde{d}>1$ and limiting case $d-\tilde{d}=1$ and show the existence of KAM tori for DNLS and perturbed BO equations.

If the Hamiltonian operator $A$ has continuous spectra, there is usually no KAM tori for the HPDE. Therefore, we should assume that the Hamiltonian operator $A$ is of pure point spectra before stating our theorems. Taking the eigenfunctions of $A$ as a basis and changing partial coordinates into actionangle variables, from the HPDE one can usually obtain a small perturbation $H=N+P$ of an infinite dimensional Hamiltonian in the parameter dependent normal form

$$
N=\sum_{1 \leq j \leq n} \omega_{j}(\xi) y_{j}+\frac{1}{2} \sum_{j \geq 1} \Omega_{j}(\xi)\left(u_{j}^{2}+v_{j}^{2}\right)
$$

on a phase space

$$
\mathscr{P}^{a, p}=\mathbb{T}^{n} \times \mathbb{R}^{n} \times \ell^{a, p} \times \ell^{a, p} \ni(x, y, u, v)
$$

with symplectic structure $\sum_{1 \leq j \leq n} d y_{j} \wedge d x_{j}+\sum_{j \geq 1} d u_{j} \wedge d v_{j}$, where $\ell^{a, p}$ is the Hilbert space of all real sequences $w=\left(w_{1}, w_{2}, \cdots\right)$ with

$$
\|w\|_{a, p}^{2}=\sum_{j \geq 1} e^{2 a j} j^{2 p}\left|w_{j}\right|^{2}<\infty
$$

where $a \geq 0$ and $p \geq 0$. The tangential frequencies $\omega=\left(\omega_{1}, \cdots, \omega_{n}\right)$ and normal frequencies $\Omega=$ $\left(\Omega_{1}, \Omega_{2}, \cdots\right)$ are real vectors depending on parameters $\xi \in \Pi \subset \mathbb{R}^{n}, \Pi$ a closed bounded set of positive Lebesgue measure, and roughly

$$
\Omega_{j}(\xi)=j^{d}+\cdots
$$

The perturbation term $P$ is real analytic in the space coordinates and Lipschitz in the parameters, and for each $\xi \in \Pi$ its Hamiltonian vector field $X_{P}=\left(P_{y},-P_{x},-P_{v}, P_{u}\right)^{T}$ defines near $\mathscr{T}_{0}:=\mathbb{T}^{n} \times$ $\{y=0\} \times\{u=0\} \times\{v=0\}$ a real analytic map

$$
X_{P}: \mathscr{P}^{a, p} \rightarrow \mathscr{P}^{a, q}
$$

where

$$
p-q=\tilde{d}
$$


In the whole of this paper the parameter $a$ is fixed. Moreover, throughout this paper, for convenience, we will adopt lots of notations and definitions from [10].

We denote by $\mathscr{P}_{\mathbb{C}}^{a, p}$ the complexification of $\mathscr{P}^{a, p}$. For $s, r>0$, We introduce the complex $\mathscr{T}_{0^{-}}$ neighborhoods in $\mathscr{P}_{\mathbb{C}}^{a, p}$

$$
D(s, r): \quad|\operatorname{Im} x|<s, \quad|y|<r^{2}, \quad\|u\|_{a, p}+\|v\|_{a, p}<r,
$$

and weighted norm for $W=(X, Y, U, V) \in \mathscr{P}_{\mathbb{C}}^{a, q}$

$$
\|W\|_{r, a, q}=|X|+\frac{|Y|}{r^{2}}+\frac{\|U\|_{a, q}}{r}+\frac{\|V\|_{a, q}}{r}
$$

where $|\cdot|$ denotes the sup-norm for complex vectors. Furthermore, for a map $W: D(s, r) \times \Pi \rightarrow \mathscr{P}_{\mathbb{C}}^{a, q}$, for example, the Hamiltonian vector field $X_{P}$, we define the norms

$$
\begin{gathered}
\|W\|_{r, a, q, D(s, r) \times \Pi}=\sup _{D(s, r) \times \Pi}\|W\|_{r, a, q}, \\
\|W\|_{r, a, q, D(s, r) \times \Pi} \operatorname{lip}_{\xi, \zeta \in \Pi, \xi \neq \zeta D(s, r)} \frac{\|\Delta \xi \zeta W\|_{r, a, q}}{|\xi-\zeta|},
\end{gathered}
$$

where $\Delta_{\xi \zeta} W=W(\cdot ; \xi)-W(\cdot ; \zeta)$. In a completely analogous manner, the Lipschitz semi-norm of the frequencies $\omega$ and $\Omega$ are defined as

$$
|\omega|_{\Pi}^{\operatorname{lip}}=\sup _{\xi, \zeta \in \Pi, \xi \neq \zeta} \frac{\left|\Delta_{\xi \zeta} \omega\right|}{|\xi-\zeta|}, \quad|\Omega|_{-\delta, \Pi}^{\operatorname{lip}}=\sup _{\xi, \zeta \in \Pi, \xi \neq \zeta j \geq 1} \sup _{j=1} \frac{j^{-\delta}\left|\Delta_{\xi \zeta} \Omega_{j}\right|}{|\xi-\zeta|}
$$

for any real number $\delta$.

Theorem 1.1. Suppose the normal form $N$ described above satisfies the following assumptions:

(A) The map $\xi \mapsto \omega(\xi)$ between $\Pi$ and its image is a homeomorphism which is Lipschitz continuous in both directions, i.e. there exist positive constants $M_{1}$ and $L$ such that $|\omega|_{\Pi}^{\operatorname{lip}} \leq M_{1}$ and $\left|\omega^{-1}\right|_{\omega(\Pi)}^{\operatorname{lip}} \leq L$;

(B) There exists $d>1$ such that

$$
\left|\Omega_{i}-\Omega_{j}\right| \geq m\left|i^{d}-j^{d}\right|
$$

for all $i \neq j \geq 0$ uniformly on $\Pi$ with some constant $m>0$. Here $\Omega_{0}=0$;

(C) There exists $\delta \leq d-1$ such that the functions $\xi \mapsto \frac{\Omega_{j}(\xi)}{j^{\delta}}$ are uniformly Lipschitz on $\Pi$ for $j \geq 1$, i.e. there exist a positive constant $M_{2}$ such that $|\Omega|_{-\delta, \Pi}^{\operatorname{lip}_{\Pi}} \leq M_{2} ;$

(D) We additionally assume

$$
4 E L M_{2} \leq m,
$$

where $E=|\omega|_{\Pi}:=\sup _{\xi \in \Pi}|\omega(\xi)|$.

Set $M=M_{1}+M_{2}$. Then for every $\beta>0$, there exists a positive constant $\gamma$, depending only on $n, d, \delta, m$, the frequencies $\omega$ and $\Omega, s>0$ and $\beta$, such that for every perturbation term $P$ described above with

$$
\tilde{d}=p-q \leq d-1
$$

and

$$
\varepsilon:=\left\|X_{P}\right\|_{r, a, q, D(s, r) \times \Pi}+\frac{\alpha}{M}\left\|X_{P}\right\|_{r, a, q, D(s, r) \times \Pi}^{\text {lip }} \leq(\alpha \gamma)^{1+\beta}
$$

for some $r>0$ and $0<\alpha<1$, there exist 
(1) a Cantor set $\Pi_{\alpha} \subset \Pi$ with

$$
\left|\Pi \backslash \Pi_{\alpha}\right| \leq c_{1} \alpha,
$$

where $|\cdot|$ denotes Lebesgue measure and $c_{1}>0$ is a constant depends on $n, \omega$ and $\Omega$;

(2) a Lipschitz family of smooth torus embeddings $\Phi: \mathbb{T}^{n} \times \Pi_{\alpha} \rightarrow \mathscr{P}^{a, p}$ satisfying: for every non-negative integer multi-index $k=\left(k_{1}, \ldots, k_{n}\right)$,

$$
\left\|\partial_{x}^{k}\left(\Phi-\Phi_{0}\right)\right\|_{r, a, p, \mathbb{T}^{n} \times \Pi_{\alpha}}+\frac{\alpha}{M}\left\|\partial_{x}^{k}\left(\Phi-\Phi_{0}\right)\right\|_{r, a, p, \mathbb{T}^{n} \times \Pi_{\alpha}}^{\text {lip }} \leq c_{2} \varepsilon^{\frac{1}{1+\beta}} / \alpha
$$

where $\partial_{x}^{k}:=\frac{\partial^{|k|}}{\partial x_{1}^{k_{1}} \cdots \partial x_{n}^{k_{n}}}$ with $|k|:=\left|k_{1}\right|+\cdots+\left|k_{n}\right|$,

$$
\Phi_{0}: \mathbb{T}^{n} \times \Pi \rightarrow \mathscr{T}_{0}, \quad(x, \xi) \mapsto(x, 0,0,0)
$$

is the trivial embedding for each $\xi$, and $c_{2}$ is a positive constant which depends on $k$ and the same parameters as $\gamma$;

(3) a Lipschitz map $\phi: \Pi_{\alpha} \rightarrow \mathbb{R}^{n}$ with

$$
|\phi-\omega|_{\Pi_{\alpha}}+\frac{\alpha}{M}|\phi-\omega|_{\Pi_{\alpha}}^{\text {lip }} \leq c_{3} \varepsilon,
$$

where $c_{3}$ is a positive constant which depends on the same parameters as $\gamma$,

such that for each $\xi \in \Pi_{\alpha}$ the map $\Phi$ restricted to $\mathbb{T}^{n} \times\{\xi\}$ is a smooth embedding of a rotational torus with frequencies $\phi(\xi)$ for the perturbed Hamiltonian $H$ at $\xi$. In other words,

$$
t \mapsto \Phi(\theta+t \phi(\xi), \xi), \quad t \in \mathbb{R}
$$

is a smooth quasi-periodic solution for the Hamiltonian $H$ evaluated at $\xi$ for every $\theta \in \mathbb{T}^{n}$ and $\xi \in \Pi_{\alpha}$.

This theorem can be applied to the class of derivative nonlinear Schrödinger equations mentioned above. However, the assumption (1.5) excludes the perturbed Benjamin-Ono equation mentioned above. Thus, in the following, we give a modified version of the above theorem:

Theorem 1.2. The above theorem also holds true with, respectively, replacing the assumption (D) and conclusion (1) by the assumption $\left(D^{*}\right)$ and conclusion $\left(1^{*}\right)$ below:

(D*) For every $k \in \mathbb{Z}^{n}$ and $l \in \mathbb{Z}^{\infty}$ with $1 \leq|l| \leq 2$ (here $|l|=\sum_{j \geq 1}\left|l_{j}\right|$ ), the resonance set

$$
\{\xi \in \Pi:\langle k, \omega(\xi)\rangle+\langle l, \Omega(\xi)\rangle=0\}
$$

has Lebesgue measure zero; Moreover, if $\delta=d-1$, we additively assume that there exist $\delta_{0}<d-1$, a partition $\Omega=\Omega^{1}+\Omega^{2}$, positive constant $M_{3}$ and $M_{4}$ with

$$
8 E L M_{4} \leq m,
$$

such that $\left|\Omega^{1}\right|_{-\delta_{0}, \Pi}^{\text {lip }} \leq M_{3},\left|\Omega^{2}\right|_{-\delta, \Pi}^{\text {lip }} \leq M_{4} ;$

(1*) a Cantor set $\Pi_{\alpha} \subset \Pi$ with $\left|\Pi \backslash \Pi_{\alpha}\right| \rightarrow 0$ as $\alpha \rightarrow 0$, where $|\cdot|$ denotes Lebesgue measure.

This paper is organized as follows: In section 2 we give an outline of the proof of the above theorems, and some new difficult and ideas compared with [7] [10] are exhibited. In sections 3-6 the above theorems are proved in detail. The proof of Theorem 1.2 is the same as that of Theorem 1.1 except the measure estimate. Thus, sections 3-5 and subsection 6.1 are devoted to the proof of Theorem 1.1, while the measure estimate for Theorem 1.2 is given in subsection 6.2. In sections 7-8 Theorem 1.1 and Theorem 1.2 are applied to derivative nonlinear Schrödinger equations and perturbed Benjamin-Ono equation, respectively. Finally, a technical lemma is listed in section 9. 


\section{Outline of The Proof and more Remarks}

The above theorems generalize Kukisn's theorem from $\tilde{d}<d-1$ to $\tilde{d} \leq d-1$ such that the range of application is extended to a class of derivative nonlinear Schrödinger equations and perturbed Benjamin-Ono equation. Here we would like to compare the proof of our theorems with that of Kuksin's theorem. By and large, as any KAM theorem, in both cases Newton iteration is used to overcome the notorious small divisor difficult. Therefore, our proof is mainly based on Kuksin's approach in [7]. (Also see [10]). There is, however, some essential differences between the proof of our theorems and that of Kuksin's theorem. In order to see clearly the differences, let us give the basic procedure of the proof of KAM theorem from [7] and [10], which consists of the following steps.

2.1. Derivation of the homological equations. For convenience, introduce complex variables $z=(u-\mathbf{i} v) / \sqrt{2}$ and $\bar{z}=(u+\mathbf{i} v) / \sqrt{2}$. Assume we are now in the $v$-th KAM iterative step. Write the integrable part $N_{v}$ of the Hamiltonian $H_{v}$

$$
N_{v}=\langle\omega, y\rangle+\sum_{j=1}^{\infty} \Omega_{j} z_{j} \bar{z}_{j}
$$

and develop the perturbation $P_{v}$ into Taylor series in $(y, z, \bar{z})$ :

$$
P_{V}=\varepsilon_{v} R_{v}+O\left(|y|^{2}+|y||| z||_{a, p}+\|z\|_{a, p}^{3}\right),
$$

where $\varepsilon_{v}$ goes to zero very fast, for example, taking $\varepsilon_{v} \approx \varepsilon^{(5 / 4)^{v}}$, and

$$
R_{v}=R^{x}(x)+\left\langle R^{y}, y\right\rangle+\left\langle R^{z}(x), z\right\rangle+\left\langle R^{\bar{z}}(x), \bar{z}\right\rangle+\left\langle R^{z z}(x) z, z\right\rangle+\left\langle R^{\bar{z} \bar{z}}(x) \bar{z}, \bar{z}\right\rangle+\left\langle R^{z \bar{z}}(x) z, \bar{z}\right\rangle=O(1) .
$$

A key point is, very roughly speaking, to search for a Hamiltonian function of the same form as $R_{V}$ :

$$
F_{v}=F^{x}(x)+\left\langle F^{y}, y\right\rangle+\left\langle F^{z}(x), z\right\rangle+\left\langle F^{\bar{z}}(x), \bar{z}\right\rangle+\left\langle F^{z z}(x) z, z\right\rangle+\left\langle F^{\bar{z} \bar{z}}(x) \bar{z}, \bar{z}\right\rangle+\left\langle F^{z \bar{z}}(x) z, \bar{z}\right\rangle
$$

which satisfies

$$
\left\{N_{v}, F_{v}\right\}+R_{v}=0,
$$

where $\{\cdot, \cdot\}$ is the Poisson bracket with respect to the symplectic structure

$$
\sum_{1 \leq j \leq n} d y_{j} \wedge d x_{j}-\mathbf{i} \sum_{j \geq 1} d z_{j} \wedge d \bar{z}_{j}
$$

By $\Phi_{v}:=X_{\varepsilon_{v} F_{v}}^{1}$ denote the time-1 map of the Hamiltonian vector field $X_{\varepsilon_{v} F_{v}}$. It is a symplectic transformation. A simple calculation shows that $\Phi_{v}$ changes $H_{v}=N_{v}+P_{v}$ into

$$
H_{v+1}=H_{v} \circ \Phi_{v}=N_{v}+\tilde{R}_{v+1}+O\left(|y|^{2}+|y||| z||_{a, p}+\|z\|_{a, p}^{3}\right)
$$

with

$$
\tilde{R}_{v+1}=\frac{1}{2} \varepsilon_{v}^{2}\left\{\left\{N_{v}, F_{v}\right\}, F_{v}\right\}+\varepsilon_{v}^{2}\left\{R_{v}, F_{v}\right\}+\cdots
$$

Our task is now to search for $F_{v}$ satisfying $\left\{N_{v}, F_{v}\right\}+R_{v}=0$ which is a set of the first order partial differential equations:

$$
\begin{gathered}
\omega \cdot \partial_{x} F^{x}(x)=R^{x}(x), \quad \omega \cdot \partial_{x} F^{y}(x)=R^{y}(x), \\
\cdots \\
\omega \cdot \partial_{x} F^{z \bar{z}}(x)+\mathbf{i} \Lambda F^{z \bar{z}}(x)-\mathbf{i} F^{z \bar{z}}(x) \Lambda=R^{z \bar{z}}(x),
\end{gathered}
$$


where $\Lambda=\operatorname{diag}\left(\Omega_{j}: j=1,2, \ldots\right)$. Let us consider the last equation which is the most difficulty one. By $F_{i j}(x)$ and $R_{i j}(x)$, denote the matrix elements of the operators $F^{z \bar{z}}$ and $R^{z \bar{z}}$, respectively. Then the last equation becomes

$$
-\mathbf{i} \omega \cdot \partial_{x} F_{i j}(x)+\left(\Omega_{i}-\Omega_{j}\right) F_{i j}(x)=-\mathbf{i} R_{i j}(x), i, j=1,2, \ldots
$$

or

$$
\left(\langle k, \omega\rangle+\Omega_{i}-\Omega_{j}\right) \widehat{F}_{i j}(k)=-\mathbf{i} \widehat{R}_{i j}(k),
$$

where $\widehat{F}_{i j}(k)$ and $\widehat{R}_{i j}(k)$ are the $k$-th Fourier coefficients of $F_{i j}(x)$ and $R_{i j}(x)$, respectively. One can assume $\widehat{R}_{j j}(0)=0$, otherwise $\widehat{R}_{j j}(0)$ is put into $\Omega_{j}$ as a modification of the normal form $N_{v}$. Thus, (2.3) can be solved by

$$
\widehat{F}_{i j}(k)=\frac{\widehat{R}_{i j}(k)}{\langle k, \omega\rangle+\Omega_{i}-\Omega_{j}}
$$

under the non-resonant conditions

$$
\langle k, \omega\rangle+\Omega_{i}-\Omega_{j} \neq 0 \quad \text { unless } \quad k=0, i=j .
$$

This is actually the KAM iterative procedure for bounded perturbation $P$. However, the thing is not so simple when the perturbation $P$ is unbounded, i.e., $\tilde{d}=p-q>0$. When

$$
X_{P}: \mathscr{P}^{a, p} \rightarrow \mathscr{P}^{a, q}, \quad \tilde{d}=p-q>0,
$$

one has, very roughly,

$$
\left|\widehat{R}_{i j}(k)\right| \approx|i|^{\tilde{d}}+|j|^{\tilde{d}} \rightarrow \infty, \quad \text { as }|i|+|j| \rightarrow \infty .
$$

This leads usually to $\widehat{F}_{i j}(k) \rightarrow \infty$. In other words, the solution $F_{v}$ or the transformation $\Phi_{v}=X_{\varepsilon_{v} F_{v}}^{1}$ would be unbounded. One should note that the coordinate transformations $\Phi_{v}=X_{\varepsilon_{v} F_{v}}^{1}$ must be bounded even if the the perturbation $P$ is unbounded in order that the domains of the KAM iteration are always in the same phase space $\mathscr{P}^{a, p}$ and that the KAM iterative procedure can work. In order to guarantee the bounded-ness of $F_{v}$, it is required in [7] and [10] that

$$
\left|\Omega_{i}-\Omega_{j}\right| \approx|i|^{d-1}+|j|^{d-1}, \quad i \neq j
$$

or rather roughly,

$$
\left|\langle k, \omega\rangle+\Omega_{i}-\Omega_{j}\right| \approx|i|^{d-1}+|j|^{d-1} .
$$

Together with $\tilde{d} \leq d-1$, one has that for $i \neq j$,

$$
\left|\widehat{F}_{i j}(k)\right| \approx \frac{|i|^{\tilde{d}}+|j|^{\tilde{d}}}{|i|^{d-1}+|j|^{d-1}}=O(1) .
$$

It is clear that this estimate fails when $i=j$. To avoid this plight, Kuksin[7] smartly put the whole $R_{j j}(x)$ rather than $\widehat{R}_{j j}(0)$ into $\Omega_{j}$ as a modification of the normal form $N_{v}$ so that it is not necessary to solve the equation for $F_{j j}(x)$. In doing so, the term $N_{v}$ becomes into the generalized normal form (i.e. normal frequencies depend on the angle variable $x$ )

$$
\tilde{N}_{v}:=N_{v}+\sum_{j=1}^{\infty} R_{j j}(x) z_{j} \bar{z}_{j}=\langle\omega, y\rangle+\sum_{j=1}^{\infty}\left(\Omega_{j}+R_{j j}(x)\right) z_{j} \bar{z}_{j},
$$

the homological equation (2.1) is modified into

$$
\left\{\tilde{N}_{v}, F_{v}\right\}+R_{v}=0,
$$


and the remaining term $\tilde{R}_{v+1}$ is changed into

$$
\tilde{R}_{v+1}=\frac{1}{2} \varepsilon_{v}^{2}\left\{\left\{\tilde{N}_{v}, F_{v}\right\}, F_{v}\right\}+\varepsilon_{v}^{2}\left\{R_{v}, F_{v}\right\}+\cdots
$$

Accordingly, the homological equation (2.3) becomes into

$$
-\mathbf{i} \omega \cdot \partial_{x} F_{i j}(x)+\left(\Omega_{i}-\Omega_{j}+R_{i i}(x)-R_{j j}(x)\right) F_{i j}(x)=-\mathbf{i} R_{i j}(x), \quad i \neq j .
$$

Let $u=F_{i j}(x), \lambda=\Omega_{i}-\Omega_{j}, \mu(x)=R_{i i}(x)-R_{j j}(x)$ and $r(x)=-\mathbf{i} R_{i j}(x)$. Then this equation can be abbreviated as an abstract equation

$$
-\mathbf{i} \omega \cdot \partial_{x} u+(\lambda+\mu(x)) u=r(x) .
$$

Since $R_{i i}$ and $R_{j j}$ are large, $\mu(x)$ is usually large. And the coefficient $\mu(x)$ involves the angle variable. The equation of this type is called "small-denominators equations with large variable coefficients" by Kuksin [6]. Remark that, for simplicity, the modification of $\omega$ is omitted here.

2.2. Solving the homological equations. In order to make KAM iterative procedure work, the existence domain of the solution $u$ should be the strip-type neighborhood of $\mathbb{T}^{n}$ with some width $s>0: D(s):=\left\{x \in \mathbb{C}^{n} / 2 \pi \mathbb{Z}^{n}:|\operatorname{Im} x| \leq s\right\}$. Assume

$$
\mu(x) \approx C \tilde{\gamma}
$$

where $C$ is some small constant and where $\tilde{\gamma}$ should be usually a large magnitude. Since (2.7) is scalar, it can be solved directly and estimated by

$$
\sup _{x \in D(s-\sigma)}|u(x)| \lesssim e^{2 C \tilde{\gamma}}\|r\|_{s}, \quad 0 \leq \sigma<s
$$

where $\|r\|_{s}:=\sup _{x \in D(s)}|r(x)|$. This estimate is, however, not good enough to support the KAM iteration procedure, since the solution $u$ becomes too large as $\mu$ is large. In fact, in the $v$-th KAM iteration step, $\tilde{\gamma} \approx 2^{v}$. Thus, $u \approx \exp \left(2^{v}\right)$ goes to infinity very rapidly, which makes the coordinate transformations essentially unbounded. When $\tilde{d}<d-1$, Kuksin's lemma [6] can solve this problem. Following Kuksin [6], we assume there are constant $C>0$ and $0<\theta<1$ such that

$$
|\lambda|^{\theta} \geq C\|\mu\|_{s}
$$

Kuksin's Lemma states that under suitable non-resonant conditions on $\omega$, the solution $u$ satisfies the estimate:

$$
\|u\|_{s-\sigma} \leq C_{1} \exp \left(C_{2} C_{3}^{\frac{1}{1-\theta}}\right)\|r\|_{s}
$$

where $C_{1}$ and $C_{2}$ are positive constants depending on only $n$ and $\sigma$, and $C_{3}>0$ is a constant depending on the non-resonant conditions. When this estimate is applied to (2.6), one needs to take

$$
\lambda=\Omega_{i}-\Omega_{j} \approx i^{d}-j^{d} \approx i^{d-1}+j^{d-1}, \quad \tilde{\gamma} \approx i^{\tilde{d}}+j^{\tilde{d}}, \quad i, j=1,2,3, \ldots
$$

From this, we see that there is indeed a constant $0<\theta<1$ such that (2.9) holds true if $\tilde{d}<d-1$. Therefore, the solution $u$ of the homological equation (2.7) has a uniform bound independent of the size of $\mu$. This makes the coordinate transformation bounded. We see also that $\tilde{d}=d-1$ leads to $\theta=1$ in (2.9). In this case, the estimate (2.10) is invalid. (The right hand side of (2.10) is equal to $\infty$.) Now it is clear that we need some new estimate for the solution $u$ covering not only the case $\tilde{d}<d-1$ but also the limit case $\tilde{d}=d-1$. The new estimate has been obtained in our recent paper [12]:

$$
\|u\|_{s-\sigma} \leq C_{4} e^{C \tilde{\gamma} s}\|r\|_{s}
$$


where $C_{4}$ is a constant depending the non-resonant conditions on $\omega$ and where $C$ is a positive constant small enough. Since the parameter $\tilde{\gamma}$ (which measures the magnitude of the perturbation $\mu(x)$ ) goes into the exponential in the right hand side of (2.11), in this sense, the upper bound of this new estimate looks weaker than that of the original Kuksin's lemma. However, compared with (2.8), there is some essential improvement in (2.11): there is an $s$ in the exponential in (2.11). This number $s$ will be used crucially in the following manner: In the $v$-th KAM iteration, we let $s=2^{-v}$. One will find that there is no the small divisor problem in the homological equation (2.7) when $\tilde{\gamma}>$ some large constant $K \approx 2^{v}(5 / 4)^{v}|\ln \varepsilon|$. In this case, the homological equation (2.7) can be solved by the implicit function theorem. So the non-trivial case is when $\tilde{\gamma} \leq K$. At this time, we find that

$$
e^{C \tilde{\gamma} s} \lesssim \varepsilon_{v}^{-C}
$$

with $\varepsilon_{v}:=\varepsilon^{(5 / 4)^{v}}$ and the constant $C \ll 1$. Thus, by the new estimate (2.11) and noting $\|r\|_{s} \leq \varepsilon_{v}$, we have

$$
\|u\|_{s-\sigma} \lesssim \varepsilon_{v}^{-C}\|r\|_{s} \leq \varepsilon_{v}^{-C} \varepsilon_{v} \lesssim \varepsilon_{v}
$$

In the usual KAM iteration, one would have obtained $\|u\|_{s-\sigma} \leq 2^{v} \varepsilon_{v} \lesssim \varepsilon_{v}$. Although here $\varepsilon_{v}^{-C} \varepsilon_{v} \gg$ $2^{v} \varepsilon_{v}$, inequality (2.12) can guarantee the KAM procedure to be iterated. Therefore, although the new estimate (2.11) is weaker than that of the original Kuksin's Lemma, it can covers both $\tilde{d}<d-1$ and the limit case $\tilde{d}=d-1$, and it is sufficient for the proof of the KAM theorems of the present paper.

2.3. Estimate of the remaining terms $\tilde{R}_{v+1}$, etc. Recall $R_{v}=O(1)$. By $(2.11), F_{v}=O\left(\varepsilon_{v}^{-C}\right)$ with $0<C \ll 1$. Thus,

$$
\left\{F_{v}, R_{v}\right\}=O\left(\varepsilon_{v}^{-C}\right) .
$$

By (2.4),

$$
\left\{\tilde{N}_{V}, F_{V}\right\}=-R_{V}=O(1) .
$$

Thus,

$$
\left\{\left\{\tilde{N}_{v}, F_{v}\right\}, F_{v}\right\}=\left\{O(1), O\left(\varepsilon_{v}^{-C}\right)\right\}=O\left(\varepsilon_{v}^{-C}\right) .
$$

Consequently, by (2.5), very roughly,

$$
\tilde{R}_{v+1}=\frac{1}{2} \varepsilon_{v}^{2}\left\{\left\{\tilde{N}, F_{v}\right\}, F_{v}\right\}+\varepsilon_{v}^{2}\left\{R_{v}, F_{v}\right\}+\cdots=O\left(\varepsilon_{v}^{2-C}\right)=O\left(\varepsilon_{v+1}\right):=\varepsilon_{v+1} R_{v+1},
$$

where $R_{v+1}=O(1)$. Therefore, we can rewrite $H_{v+1}$ as

$$
H_{v+1}=\tilde{N}_{v+1}+\varepsilon_{v+1} R_{v+1}+O\left(|y|^{2}+|y||| z||_{a, p}+\|z\|_{a, p}^{3}\right) .
$$

2.4. Convergence of the iterative procedure. Repeating the above procedure and letting $v \rightarrow \infty$ and noting $\varepsilon_{v} \rightarrow 0$ very fast, one can finally get

$$
H_{\infty}:=\lim _{v \rightarrow \infty} H \circ \Phi_{1} \circ \cdots \circ \Phi_{v}=\tilde{N}_{\infty}+O\left(|y|^{2}+|y||| z\left\|_{a, p}+\right\| z \|_{a, p}^{3}\right),
$$

where $\tilde{N}_{\infty}=\lim _{v \rightarrow \infty} \tilde{N}_{v}=\left\langle\omega_{\infty}, y\right\rangle+\sum_{j \geq 1} \Omega_{\infty, j}(x) z_{j} \bar{z}_{j}$. The system corresponding to $H_{\infty}$ is

$$
\left\{\begin{array}{l}
\dot{x}=\frac{\partial H_{\infty}}{\partial y}=\omega_{\infty}+O\left(|y|+\|z\|_{a, p}\right) \\
\dot{y}=-\frac{\partial H_{\infty}}{\partial x}=O\left(|y|^{2}+|y||| z\left\|_{a, p}+\right\| z \|_{a, p}^{2}\right) \\
\dot{z}_{j}=-\mathbf{i} \frac{\partial H_{\infty}}{\partial \bar{z}_{j}}=-\mathbf{i} \Omega_{\infty, j} z_{j}+O\left(|y|+\|z\|_{a, p}^{2}\right), \quad j \geq 1 .
\end{array}\right.
$$

It is clearly seen that

$$
\mathscr{T}_{\infty}:=\left\{(x, y, z, \bar{z}) \in \mathscr{P}^{a, p}: x=\omega_{\infty} t, y=0, z=\bar{z}=0, t \in \mathbb{R}\right\}
$$


forms an invariant torus of the hamiltonian vector field $X_{H_{\infty}}$. Going back to the original vector field $X_{H}$, then $\left(\lim _{v \rightarrow \infty} \Phi_{1} \circ \cdots \circ \Phi_{v}\right) \mathscr{T}_{\infty}$ is an invariant torus of the Hamiltonian system defined by $H$.

2.5. Estimate of the measure of the parameters. When $\tilde{d}=d-1$, another new difficult also arises in search for $F$. It is under suitable non-resonant conditions that either (2.11) or (2.10) can hold true. In other words, one has to remove some resonant sets consisting of "bad" parameters $\xi$, equivalently, to remove the "bad" parameters $\omega$ when $\omega=\omega(\xi)$ depends on $\xi$ in some non-degenerate way. For example, we need to eliminate the resonant set

$$
\left\{\xi \in \Pi:\langle k, \omega(\xi)\rangle+\Omega_{i}(\xi)-\Omega_{j}(\xi) \text { is small }\right\}
$$

where $i \neq j \in \mathbb{N}$ and $k \in \mathbb{Z}^{n}$. Clearly we hope that the Lebesgue measure of the set is small. To that end we need to verify that $\langle k, \omega(\xi)\rangle+\Omega_{i}(\xi)-\Omega_{j}(\xi)$ is twisted with respect to $\xi \in \Pi$, equivalently, twisted with respect to $\omega \in \omega(\Pi)$, that is, we need to show

$$
(\star):=\left|\langle k, \omega\rangle+\Omega_{i}(\xi(\omega))-\Omega_{j}(\xi(\omega))\right|_{\omega(\Pi)}^{\text {lip }}>0,
$$

where $\omega(\xi(\omega))=\omega$.

Recall $\left|\Omega_{i}-\Omega_{j}\right| \geq m\left|i^{d}-j^{d}\right|$. Thus, $\langle k, \omega\rangle+\Omega_{i}-\Omega_{j}$ is not small if $|k| \leq \tilde{C}_{0}\left|i^{d}-j^{d}\right|$ with some constant $\tilde{C}_{0}$ depending on $m$ and $|\omega|_{\Pi}$. Now we assume $|k| \geq \tilde{C}_{0}\left|i^{d}-j^{d}\right|$. At the $v$-th KAM step, because of the modification of frequencies from unbounded perturbation, we have

$$
\left|\Omega_{j}\right|_{\omega(\Pi)}^{\text {lip }}=O\left(j^{\delta}\right)+O\left(j^{\tilde{d}}\right) .
$$

By a small trick (See $\S 3.2$ below.), we can let $\delta=\tilde{d}$. Therefore, there exists a constant $\tilde{C}_{1}$ such that

$$
\begin{aligned}
(\star) & \geq|k|-\tilde{C}_{1}\left(i^{\tilde{d}}+j^{\tilde{d}}\right) \\
& \geq \frac{1}{2} \tilde{C}_{0}\left(i^{d-1}+j^{d-1}\right)-\tilde{C}_{1}\left(i^{\tilde{d}}+j^{\tilde{d}}\right) \\
& \geq\left(\frac{1}{4} \tilde{C}_{0}\left(i^{d-1-\tilde{d}}+j^{d-1-\bar{d}}\right)-\tilde{C}_{1}\right)\left(i^{\tilde{d}}+j^{\tilde{d}}\right),
\end{aligned}
$$

which is similar to (10.50) in [7] (See also page 174 in [10]). When $\tilde{d}<d-1$, it follows from (2.16) that $(\star)>0$ if

$$
\max (i, j)>\left(\frac{4 \tilde{C}_{1}}{\tilde{C}_{0}}\right)^{\frac{1}{d-1-d}}:=\mathscr{K} .
$$

Therefore, if $\tilde{d}<d-1$ it remains to verify the twist condition $(\star)>0$ just for only finite number of cases

$$
(k, i, j): \quad \tilde{C}_{0}\left|i^{d}-j^{d}\right| \leq|k| \leq \tilde{C}_{1}\left(i^{\bar{d}}+j^{\tilde{d}}\right) \quad \text { and } \quad i, j \leq \mathscr{K} .
$$

Note that the constant $\mathscr{K}$ is independent of $i, j$ and $k$, so is the number of the cases. For these cases, the measure estimate of the resonant sets can be dealt with by some initial assumptions (For example, see Proposition 22.2 in [10]). When $\tilde{d}=d-1, \mathscr{K}=+\infty$. However, it follows directly from (2.15) that $(\star)>\left(\frac{1}{2} \tilde{C}_{0}-\tilde{C}_{1}\right)\left(i^{d-1}+j^{d-1}\right)>0$ if $\tilde{C}_{0}>2 \tilde{C}_{1}$. This completes the measure estimate of the resonant sets for Theorem 1.1. One can verify that the condition $\tilde{C}_{0}>2 \tilde{C}_{1}$ is indeed satisfied by DNLS equations.

However, the inequality $\tilde{C}_{0}>2 \tilde{C}_{1}$ is not satisfied by BO equation. The procedure of the measure estimate of the resonant set is modified as follows:

Assume that $\Omega$ can be split into two part: $\Omega_{j}=\Omega_{j}^{1}+\Omega_{j}^{2}$ such that $\left|\Omega_{j}^{1}\right|_{\omega(\Pi)}^{\text {lip }} \leq \tilde{C}_{2} j^{\delta_{0}}$ with $\delta_{0}<$ $d-1$, and $\left|\Omega_{j}^{2}\right|_{\omega(\Pi)}^{\operatorname{lip}} \leq \tilde{C}_{3} j^{d-1}$ with $\tilde{C}_{3}$ suitably small. Thus,

$$
\begin{aligned}
(\star) & \geq\left(|k|-\tilde{C}_{2}\left(i^{\delta_{0}}+j^{\delta_{0}}\right)-\tilde{C}_{3}\left(i^{d-1}+j^{d-1}\right)\right) \\
& \geq\left(\frac{1}{2} \tilde{C}_{0}-\tilde{C}_{3}\right)\left(i^{d-1}+j^{d-1}\right)-\tilde{C}_{2}\left(i^{\delta_{0}}+j^{\delta_{0}}\right) .
\end{aligned}
$$


It follows from $\delta_{0}<d-1$ that there is a constant $\tilde{\mathscr{K}}=\tilde{\mathscr{K}}\left(d, \delta_{0}, \tilde{C}_{0}, \tilde{C}_{2}, \tilde{C}_{3}\right)>0$ such that $(\star)>0$ if $\max \{i, j\}>\tilde{\mathscr{K}}$ and $\frac{1}{2} \tilde{C}_{0}>\tilde{C}_{3}$. The latter inequality is satisfied by BO equation with $d=2$ and $\delta_{0}=0$. We also mention that the partition of $\Omega$ into $\Omega^{1}+\Omega^{2}$ is rather natural. In fact, $\Omega^{1}$ is usually regarded as the initial frequency vector, while $\Omega^{2}$ corresponds to the modification in KAM iteration steps.

Remarks. 1. In [12] we mentioned Theorem 1.1 above and its application to DNLS equation without proof. In this paper we give the proof and add Theorem 1.2 and a new application to Benjamin-Ono equation.

2. The proofs of both (2.10) and (2.11) depends heavily on the fact that the homological equation (2.7) is 1-dimensional so that the solution can be expressed explicitly. This dimensional restriction requires that the normal frequency $\Omega_{j}$ 's must be simple, i.e., $\Omega_{j}^{\sharp}=1$. Therefore, the range of application of Theorems 1.1 and 1.2 and Kuksin's KAM theorem ([7]) lies in those PDEs with simple frequencies such as DNLS equation subject to Dirichlet boundary conditions, KdV and BO equations with periodic boundary conditions. For a class of DNLS equation

$$
\mathbf{i} u_{t}+u_{x x}-M_{\sigma} u+\mathbf{i}\left(|u|^{2} u\right)_{x}=0
$$

subject to periodic boundary conditions, the multiplicity $\Omega_{j}^{\sharp}=2$. And for Kadomtsev-Petviashvili (KP) equation

$$
\left(u_{t}+u_{x x x}+u u_{x}\right)_{x} \pm u_{y y}=0, \quad u=u(t, x, y),(x, y) \in \mathbb{T}^{2},
$$

its frequency multiplicity $\Omega_{j}^{\sharp} \rightarrow \infty$ as $|j| \rightarrow \infty$. There is nothing to know about the existence of KAM tori for these two classes of PDEs with perturbations. In particular, the existence of KAM tori for $\mathrm{KP}$ equation is a well-known open problem by Kuksin. See [8],[4].

\section{The Homological Equations}

\subsection{Derivation of Homological Equations}

The proof of Theorem 1.1 employs the rapidly converging iteration scheme of Newton type to deal with small divisor problems introduced by Kolmogorov, involving infinite sequence of coordinate transformations. At the $v$-th step of the scheme, a Hamiltonian

$$
H_{v}=N_{v}+P_{v}
$$

is considered, as a small perturbation of some normal form $N_{v}$. A transformation $\Phi_{v}$ is set up so that

$$
H_{v} \circ \Phi_{v}=N_{v+1}+P_{V+1}
$$

with another normal form $N_{v+1}$ and a much smaller perturbation $P_{v+1}$. We drop the index $v$ of $H_{v}$, $N_{v}, P_{v}, \Phi_{v}$ and shorten the index $v+1$ as + .

Using convenient complex notation $z=(u-\mathbf{i} v) / \sqrt{2}$ and $\bar{z}=(u+\mathbf{i} v) / \sqrt{2}$, the generalized normal form reads

$$
N=\langle\omega(\xi), y\rangle+\sum_{j \geq 1} \Omega_{j}(x ; \xi) z_{j} \bar{z}_{j} .
$$

Let $R$ be 2-order Taylor polynomial truncation of $P$, that is,

$$
R=R^{x}+\left\langle R^{y}, y\right\rangle+\left\langle R^{z}, z\right\rangle+\left\langle R^{\bar{z}}, \bar{z}\right\rangle+\left\langle R^{z z} z, z\right\rangle+\left\langle R^{\bar{z} \bar{z}} \bar{z}, \bar{z}\right\rangle+\left\langle R^{z \bar{z}} z, \bar{z}\right\rangle,
$$

where $\langle\cdot, \cdot\rangle$ is formal product for two column vectors and $R^{x}, R^{y}, R^{z}, R^{\bar{z}}, R^{z z}, R^{\bar{z} \bar{z}}, R^{z \bar{z}}$ depend on $x$ and $\xi$. For a function $u$ on $\mathbb{T}^{n}$, let

$$
[u]=\frac{1}{(2 \pi)^{n}} \int_{\mathbb{T}^{n}} u(x) d x .
$$


By $\llbracket R \rrbracket$ denote the part of $R$ in generalized normal form as follows

$$
\llbracket R \rrbracket=\left[R^{x}\right]+\left\langle\left[R^{y}\right], y\right\rangle+\left\langle\operatorname{diag}\left(R^{z \bar{z}}\right) z, \bar{z}\right\rangle,
$$

where $\operatorname{diag}\left(R^{z \bar{z}}\right)$ is the diagonal of $R^{z \bar{z}}$. Note that $\left[R^{x}\right]$ and $\left[R^{y}\right]$ are independent of $x$. In the following, the term $\left[R^{x}\right]$ will be omitted since it does not affect the dynamics.

The coordinate transformation $\Phi$ is obtained as the time-1-map $\left.X_{F}^{t}\right|_{t=1}$ of a Hamiltonian vector field $X_{F}$, where $F$ is of the same form as $R$ :

$$
F=F^{x}+\left\langle F^{y}, y\right\rangle+\left\langle F^{z}, z\right\rangle+\left\langle F^{\bar{z}}, \bar{z}\right\rangle+\left\langle F^{z z} z, z\right\rangle+\left\langle F^{\bar{z} \bar{z}} \bar{z}, \bar{z}\right\rangle+\left\langle F^{z \bar{z}} z, \bar{z}\right\rangle,
$$

and $\llbracket F \rrbracket=0$. Denote $\partial_{\omega}=\sum_{1 \leq b \leq n} \omega_{b} \frac{\partial}{\partial x_{b}}, \Lambda=\operatorname{diag}\left(\Omega_{j}: j \geq 1\right)$. Then we have

$$
\begin{aligned}
H \circ \Phi= & (N+R) \circ X_{F}^{1}+(P-R) \circ X_{F}^{1} \\
= & N+\{N, F\}+R+\int_{0}^{1}\{(1-t)\{N, F\}+R, F\} \circ X_{F}^{t} d t+(P-R) \circ X_{F}^{1} \\
= & N+\sum_{j \geq 1}\left\langle\partial_{x} \Omega_{j}, F^{y}\right\rangle z_{j} \bar{z}_{j}+\left\langle\left[R^{y}\right], y\right\rangle+\left\langle\operatorname{diag}\left(R^{z \bar{z}}\right) z, \bar{z}\right\rangle \\
& +\left(-\partial_{\omega} F^{x}+R^{x}\right) \\
& +\left\langle-\partial_{\omega} F^{y}+R^{y}-\left[R^{y}\right], y\right\rangle \\
& +\left\langle-\partial_{\omega} F^{z}+\mathbf{i} \Lambda F^{z}+R^{z}, z\right\rangle \\
& +\left\langle-\partial_{\omega} F^{\bar{z}}-\mathbf{i} \Lambda F^{\bar{z}}+R^{\bar{z}}, \bar{z}\right\rangle \\
& +\left\langle\left(-\partial_{\omega} F^{z z}+\mathbf{i} \Lambda F^{z z}+\mathbf{i} F^{z z} \Lambda+R^{z z}\right) z, z\right\rangle \\
& +\left\langle\left(-\partial_{\omega} F^{\bar{z} \bar{z}}-\mathbf{i} \Lambda F^{\bar{z} \bar{z}}-\mathbf{i} F^{\bar{z} \bar{z}} \Lambda+R^{\bar{z} \bar{z}}\right) \bar{z}, \bar{z}\right\rangle \\
& +\left\langle\left(-\partial_{\omega} F^{z \bar{z}}-\mathbf{i} \Lambda F^{z \bar{z}}+\mathbf{i} F^{z \bar{z}} \Lambda+R^{z \bar{z}}-\operatorname{diag}\left(R^{z \bar{z}}\right)\right) z, \bar{z}\right\rangle \\
& +\int_{0}^{1}\{(1-t)\{N, F\}+R, F\} \circ X_{F}^{t} d t+(P-R) \circ X_{F}^{1} .
\end{aligned}
$$

We wish to find the function $F$ such that (3.5)-(3.11) vanish. To this end, $F^{x}, F^{y}, F^{z}, F^{\bar{z}}, F^{z z}, F^{\bar{z}}$ and $F^{z \bar{z}}$ should satisfy the homological equations:

$$
\begin{aligned}
\partial_{\omega} F^{x} & =R^{x}, \\
\partial_{\omega} F^{y} & =R^{y}-\left[R^{y}\right], \\
\partial_{\omega} F_{j}^{z}-\mathbf{i} \Omega_{j} F_{j}^{z} & =R_{j}^{z}, \quad j \geq 1, \\
\partial_{\omega} F_{j}^{\bar{z}}+\mathbf{i} \Omega_{j} F_{j}^{\bar{z}} & =R_{j}^{\bar{z}}, \quad j \geq 1, \\
\partial_{\omega} F_{i j}^{z z}-\mathbf{i}\left(\Omega_{i}+\Omega_{j}\right) F_{i j}^{z z} & =R_{i j}^{z z}, \quad i, j \geq 1, \\
\partial_{\omega} F_{i j}^{\bar{z} \bar{z}}+\mathbf{i}\left(\Omega_{i}+\Omega_{j}\right) F_{i j}^{\bar{z} \bar{z}} & =R_{i j}^{\bar{z} \bar{z}}, \quad i, j \geq 1, \\
\partial_{\omega} F_{i j}^{z \bar{z}}+\mathbf{i}\left(\Omega_{i}-\Omega_{j}\right) F_{i j}^{z \bar{z}} & =R_{i j}^{z \bar{z}}, \quad i, j \geq 1, \quad i \neq j .
\end{aligned}
$$

\subsection{Solving the Homological Equations}

Let $\Omega=\left(\Omega_{j}: j \geq 1\right), \bar{\Omega}=[\Omega]$ and $\tilde{\Omega}=\Omega-\bar{\Omega}$. Define

$$
\langle k\rangle=\max \{1,|k|\}, \quad\langle l\rangle_{d}=\max \left\{1,\left|\sum_{j \geq 1} j^{d} l_{j}\right|\right\} .
$$


Moreover, for an analytic function $u$ on $D(s)$, we define

$$
|u|_{s, \tau}:=\sum_{k \in \mathbb{Z}^{n}}\left|\hat{u}_{k}\right||k|^{\tau} e^{|k| s}
$$

where $\hat{u}_{k}:=(2 \pi)^{-n} \int_{\mathbb{T}^{n}} u(x) e^{-\mathbf{i} k \cdot x} d x$ is the $k$-Fourier coefficient of $u$.

Consider the conditions $\delta \leq d-1$ and $\tilde{d}=p-q \leq d-1$. If $\delta>\tilde{d}$, decreasing $q$ such that $\delta=\tilde{d}$, then for the new $q$, the inequality (1.7) still holds true; if $\delta<\tilde{d}$, increasing $\delta$ such that $\delta=\tilde{d}$, then for the new $\delta$, the assumption (C) still holds true, and if $\tilde{d}=d-1$, the assumption (D*) is satisfied with $\Omega_{2}=0$. Thus, without loss of generality we assume $\delta=\tilde{d} \leq d-1$ in the following.

Equations (3.13)-(3.19) will be solved under the following conditions: uniformly on $\Pi$,

$$
\begin{gathered}
|\langle k, \omega(\xi)\rangle+\langle l, \bar{\Omega}(\xi)\rangle| \geq \alpha \frac{\langle l\rangle_{d}}{\langle k\rangle \tau}, \quad k \neq 0, \quad|l| \leq 2, \\
|\langle l, \bar{\Omega}(\xi)\rangle| \geq m\langle l\rangle_{d}, \quad 0<|l| \leq 2, \\
\left|\tilde{\Omega}_{j}\right|_{s, \tau+1} \leq \alpha \gamma_{0} j^{\delta}, \quad j \geq 1,
\end{gathered}
$$

with constants $\tau \geq n, d>1,0<\gamma_{0} \leq 1 / 8, m>0$, and a parameter $0<\alpha \leq m$. We mention that $d$ is the same as in Theorem 1.1 and $\alpha, m$ will be the iteration parameters $\alpha_{v}, m_{v}$ in the $v$-th KAM step.

Equations (3.13) (3.14) can be easily solved by a standard approach in classical, finite dimensional KAM theory, so we only give the related results at the end of this subsection. Equations (3.15)-(3.18) are easier than (3.19) and can be solved in the same way as (3.19) done, so we only give the details of solving (3.19) in the following.

For any positive number $K$, we introduce a truncation operator $\Gamma_{K}$ as follows:

$$
\left(\Gamma_{K} f\right)(x):=\sum_{|k| \leq K} \hat{f}_{k} e^{\mathbf{i} k \cdot x}, \quad \forall f: \mathbb{T}^{n} \rightarrow \mathbb{C}
$$

where $\hat{f}_{k}$ is the $k$-Fourier coefficient of $f$.

Set $C_{0}=2|\omega|_{\Pi} / m$ and $K$ being a positive number which will be the iteration parameter $K_{V}$ in the $v$-th KAM step.

(1) For $(i, j)$ with $0<\left|i^{d}-j^{d}\right|<C_{0} K$, we solve exactly (3.19):

$$
\partial_{\omega} F_{i j}^{z \bar{z}}+\mathbf{i}\left(\Omega_{i}-\Omega_{j}\right) F_{i j}^{z \bar{z}}=R_{i j}^{z \bar{z}} ;
$$

(2) for $(i, j)$ with $\left|i^{d}-j^{d}\right| \geq C_{0} K$, we solve the truncated equation of (3.19):

$$
\partial_{\omega} F_{i j}^{z \bar{z}}+\mathbf{i} \Gamma_{K}\left(\left(\Omega_{i}-\Omega_{j}\right) F_{i j}^{z \bar{z}}\right)=\Gamma_{K} R_{i j}^{z \bar{z}}, \quad \Gamma_{K} F_{i j}^{z \bar{z}}=F_{i j}^{z \bar{z}} .
$$

Comparing (3.24) with (3.19), we find that (3.11) doesn't vanish. Actually, at this time, (3.11) is equivalent to $\left\langle\hat{R}^{z \bar{z}} z, \bar{z}\right\rangle$ with the matrix elements of $\hat{R}^{z \bar{z}}$ being defined by

$$
\hat{R}_{i j}^{z \bar{z}}=\left\{\begin{array}{l}
0, \quad\left|i^{d}-j^{d}\right|<C_{0} K, \\
\left(1-\Gamma_{K}\right)\left(-\mathbf{i}\left(\Omega_{i}-\Omega_{j}\right) F_{i j}^{z \bar{z}}+R_{i j}^{z \bar{z}}\right), \quad\left|i^{d}-j^{d}\right| \geq C_{0} K .
\end{array}\right.
$$

Letting $\Omega_{i j}=\Omega_{i}-\Omega_{j}=\bar{\Omega}_{i j}+\tilde{\Omega}_{i j}$ and dropping the superscript ' $z \bar{z}$ ' for brevity, (3.23) (3.24) (3.25) become

$$
-\mathbf{i} \partial_{\omega} F_{i j}+\bar{\Omega}_{i j} F_{i j}+\tilde{\Omega}_{i j} F_{i j}=-\mathbf{i} R_{i j}
$$




$$
\begin{aligned}
& -\mathbf{i} \partial_{\omega} F_{i j}+\bar{\Omega}_{i j} F_{i j}+\Gamma_{K}\left(\tilde{\Omega}_{i j} F_{i j}\right)=-\mathbf{i} \Gamma_{K} R_{i j}, \\
\hat{R}_{i j}= & \left\{\begin{array}{l}
0, \quad 0<\left|i^{d}-j^{d}\right|<C_{0} K, \\
\left(1-\Gamma_{K}\right)\left(-\mathbf{i} \tilde{\Omega}_{i j} F_{i j}+R_{i j}\right), \quad\left|i^{d}-j^{d}\right| \geq C_{0} K .
\end{array}\right.
\end{aligned}
$$

We are now in position to solve the homological equations (3.26) (3.27) by using the following two lemmas, which have been proved in [12] as Theorem 1.4 and Lemma 2.6 respectively:

Lemma 3.1 ([12]). Consider the first order partial differential equation

$$
-\mathbf{i} \partial_{\omega} u+\lambda u+\mu(x) u=p(x), \quad x \in \mathbb{T}^{n},
$$

for the unknown function $u$ defined on the torus $\mathbb{T}^{n}$, where $\omega=\left(\omega_{1}, \cdots, \omega_{n}\right) \in \mathbb{R}^{n}$ and $\lambda \in \mathbb{C}$. Assume

(1) There are constants $\alpha, \tilde{\gamma}>0$ and $\tau>n$ such that

$$
\begin{array}{r}
|k \cdot \omega| \geq \frac{\alpha}{|k|^{\tau}}, \quad k \in \mathbb{Z}^{n} \backslash\{0\}, \\
|k \cdot \omega+\lambda| \geq \frac{\alpha \tilde{\gamma}}{1+|k|^{\tau}}, \quad k \in \mathbb{Z}^{n} .
\end{array}
$$

(2) $\mu: D(s) \rightarrow \mathbb{C}$ is real analytic (here 'real' means $\mu\left(\mathbb{T}^{n}\right) \subset \mathbb{R}$ ) and is of zero average: $[\mu]=0$. Moreover, assume there is constant $C>0$ such that

$$
|\mu|_{s, \tau+1} \leq C \tilde{\gamma}
$$

(3) $p(x)$ is analytic in $x \in D(s)$.

Then (3.29) has a unique solution $u(x)$ which is defined in a narrower domain $D(s-\sigma)$ with $0<$ $\sigma<s$, and which satisfies

$$
\sup _{x \in D(s-\sigma)}|u(x)| \leq \frac{c(n, \tau)}{\alpha \gamma \sigma^{n+\tau}} e^{2 C \tilde{\gamma} s / \alpha} \sup _{x \in D(s)}|p(x)|
$$

for $0<\sigma<\min \{1, s\}$, where the constant $c(n, \tau)=(6 e+6)^{n}\left[1+\left(\frac{3 \tau}{e}\right)^{\tau}\right]$.

Lemma 3.2 ([12]). Consider the first order partial differential equation with the truncation operator $\Gamma_{K}$

$$
-\mathbf{i} \partial_{\omega} u+\lambda u+\Gamma_{K}(\mu u)=\Gamma_{K} p, \quad x \in \mathbb{T}^{n},
$$

for the unknown function $u$ defined on the torus $\mathbb{T}^{n}$, where $\omega \in \mathbb{R}^{n}, 0 \neq \lambda \in \mathbb{C}$, and $0<2 K|\omega| \leq|\lambda|$. Assume that $\mu$ is real analytic in $x \in D(s)$ with

$$
\sum_{k \in \mathbb{Z}^{n}}\left|\hat{\mu}_{k}\right| e^{|k| s} \leq \frac{|\lambda|}{4 \imath}
$$

for some constant $\imath \geq 1$, and assume $p(x)$ is analytic in $x \in D(s)$. Then (3.34) has a unique solution $u(x)$ with $u=\Gamma_{K} u$ and

$$
\begin{gathered}
\sup _{x \in D(s-\sigma)}|u(x)| \leq \frac{c(n)}{|\lambda| \sigma^{n}} \sup _{x \in D(s)}|p(x)|, \\
\sup _{x \in D(s-\sigma)}\left|\left(1-\Gamma_{K}\right)(\mu u)(x)\right| \leq \frac{c(n)}{l \sigma^{n}} e^{-9 K \sigma / 10} \sup _{x \in D(s)}|p(x)|
\end{gathered}
$$

for $0<\sigma<s$, where the constant $c(n)=4(20 e+20)^{n}$. 
Set $0<\sigma<\min \{1, s / 5\}$. In what follows the notation $a \lessdot b$ stands for "there exists a positive constant $c$ such that $a \leq c b$, where $c$ can only depend on $n, \tau$."

First, let us consider (3.26) for $(i, j)$ with $0<\left|i^{d}-j^{d}\right|<C_{0} K$. From (3.20) (3.21) we get

$$
\begin{gathered}
|\langle k, \omega(\xi)\rangle| \geq \frac{\alpha}{|k|^{\tau}}, \quad k \in \mathbb{Z}^{n} \backslash\{0\}, \\
\left|\langle k, \omega(\xi)\rangle+\bar{\Omega}_{i j}\right| \geq \frac{\alpha\left|i^{d}-j^{d}\right|}{1+|k|^{\tau}}, \quad k \in \mathbb{Z}^{n} .
\end{gathered}
$$

From (3.22) we get

$$
\left|\tilde{\Omega}_{i j}\right|_{s, \tau+1} \leq \alpha \gamma_{0}\left(i^{\delta}+j^{\delta}\right) \leq 2 \alpha \gamma_{0}\left|i^{d}-j^{d}\right| .
$$

Applying Lemma 3.1 to (3.26), we have

$$
\left|F_{i j}\right|_{D(s-2 \sigma)} \lessdot \frac{1}{\alpha\left|i^{d}-j^{d}\right| \sigma^{n+\tau}} e^{4 \gamma_{0}\left|i^{d}-j^{d}\right| s}\left|R_{i j}\right|_{D(s-\sigma)} .
$$

In view of

$$
\left|i^{d}-j^{d}\right|<C_{0} K
$$

we get

$$
\left|F_{i j}\right|_{D(s-2 \sigma)} \lessdot \frac{1}{\alpha\left|i^{d}-j^{d}\right| \sigma^{n+\tau}} e^{4 C_{0} \gamma_{0} K s}\left|R_{i j}\right|_{D(s-\sigma)} .
$$

Then, let us consider (3.27) for $(i, j)$ with $\left|i^{d}-j^{d}\right| \geq C_{0} K$. From (3.22) (3.21) we get

$$
\left|\tilde{\Omega}_{i j}\right|_{s, 0} \leq\left|\tilde{\Omega}_{i j}\right|_{s, \tau+1} \leq \alpha \gamma_{0}\left(i^{\delta}+j^{\delta}\right) \leq \frac{2 \alpha \gamma_{0}\left|\bar{\Omega}_{i j}\right|}{m|i-j|} \leq \frac{\left|\bar{\Omega}_{i j}\right|}{4|i-j|}
$$

Now applying Lemma 3.2 to (3.27), we have

$$
\begin{gathered}
\left|F_{i j}\right|_{D(s-2 \sigma)} \lessdot \frac{1}{m\left|i^{d}-j^{d}\right| \sigma^{n}}\left|R_{i j}\right|_{D(s-\sigma)}, \\
\left|\left(1-\Gamma_{K}\right)\left(\tilde{\Omega}_{i j} F_{i j}\right)\right|_{D(s-2 \sigma)} \lessdot \frac{1}{|i-j| \sigma^{n}} e^{-9 K \sigma / 10}\left|R_{i j}\right|_{D(s-\sigma)} .
\end{gathered}
$$

For a bounded linear operator from $\ell^{a, p}$ to $\ell^{a, q}$, define its operator norm by $\|\cdot\|_{a, q, p}$. As in lemma 19.1 of [10], in view of (3.42) and (3.44), using Lemma 9.1 below, we get the estimates of $F^{z \bar{z}}$ :

$$
\begin{aligned}
\left\|F^{z \bar{z}}\right\|_{a, p, p, D(s-2 \sigma)},\left\|F^{z \bar{z}}\right\|_{a, q, q, D(s-2 \sigma)} & \lessdot \frac{1}{\alpha \sigma^{2 n+\tau}} e^{4 C_{0} \gamma_{0} K s}\left\|R^{z \bar{z}}\right\|_{a, q, p, D(s)} \\
& \lessdot \frac{1}{\alpha \sigma^{2 n+\tau}} e^{4 C_{0} \gamma_{0} K s}\left\|X_{R}\right\|_{r, a, q, D(s, r)} .
\end{aligned}
$$

Multiplying by $z, \bar{z}$ we then get

$$
\frac{1}{r^{2}}\left|\left\langle F^{z \bar{z}} z, \bar{z}\right\rangle\right|_{D(s-2 \sigma, r)} \leq\left\|F^{z \bar{z}}\right\|_{a, p, p, D(s-2 \sigma)}
$$

and finally by Cauchy's estimate we have

$$
\left\|X_{\left\langle F^{\left.z \bar{z}_{z}, \bar{z}\right\rangle}\right.}\right\|_{r, a, p, D(s-3 \sigma, r)} \lessdot \frac{1}{\alpha \sigma^{2 n+\tau+1}} e^{4 C_{0} \gamma_{0} K s}\left\|X_{R}\right\|_{r, a, q, D(s, r)} .
$$


To obtain the estimate of the Lipschitz semi-norm, we proceed as follows. Shortening $\Delta_{\xi \zeta}$ as $\Delta$ and applying it to (3.26) and (3.27), one gets that, for $(i, j)$ with $0<\left|i^{d}-j^{d}\right|<C_{0} K$,

$$
\mathbf{i} \partial_{\omega}\left(\Delta F_{i j}\right)+\bar{\Omega}_{i j} \Delta F_{i j}+\tilde{\Omega}_{i j} \Delta F_{i j}=-\mathbf{i} \partial_{\Delta \omega} F_{i j}-\left(\Delta \Omega_{i j}\right) F_{i j}+\mathbf{i} \Delta R_{i j}:=Q_{i j}
$$

and that, for $(i, j)$ with $\left|i^{d}-j^{d}\right| \geq C_{0} K$,

$$
\mathbf{i} \partial_{\omega}\left(\Delta F_{i j}\right)+\bar{\Omega}_{i j} \Delta F_{i j}+\Gamma_{K}\left(\tilde{\Omega}_{i j} \Delta F_{i j}\right)=-\mathbf{i} \partial_{\Delta \omega} F_{i j}-\Gamma_{k}\left(\left(\Delta \Omega_{i j}\right) F_{i j}-\mathbf{i} \Delta R_{i j}\right):=Q_{i j}
$$

For $0<\left|i^{d}-j^{d}\right|<C_{0} K$, we have

$$
\begin{aligned}
\left|Q_{i j}\right|_{D(s-3 \sigma)} & \leq \frac{|\Delta \omega|}{\sigma}\left|F_{i j}\right|_{D(s-2 \sigma)}+\left(i^{\delta}+j^{\delta}\right)|\Delta \Omega|_{-\delta, D(s)}\left|F_{i j}\right|_{D(s-3 \sigma)}+\left|\Delta R_{i j}\right|_{D(s-3 \sigma)} \\
& \lessdot \frac{e^{4 C_{0} \gamma_{0} K s}}{\alpha \sigma^{n+\tau+1}}\left(|\Delta \omega|+|\Delta \Omega|_{-\delta, D(s)}\right)\left|R_{i j}\right|_{D(s-\sigma)}+\left|\Delta R_{i j}\right|_{D(s-\sigma)} \\
& \lessdot \frac{e^{4 C_{0} \gamma_{0} K s}}{\sigma^{n+\tau+1}}\left(\frac{|\Delta \omega|+|\Delta \Omega|_{-\delta, D(s)}}{\alpha}\left|R_{i j}\right|_{D(s-\sigma)}+\left|\Delta R_{i j}\right|_{D(s-\sigma)}\right) .
\end{aligned}
$$

Again applying Lemma 3.1 to (3.49), we have

$$
\left|\Delta F_{i j}\right|_{D(s-4 \sigma)} \lessdot \frac{e^{8 C_{0} \gamma_{0} K s}}{\alpha\left|i^{d}-j^{d}\right| \sigma^{2 n+2 \tau+1}}\left(\frac{|\Delta \omega|+|\Delta \Omega|_{-\delta, D(s)}}{\alpha}\left|R_{i j}\right|_{D(s-\sigma)}+\left|\Delta R_{i j}\right|_{D(s-\sigma)}\right) .
$$

For $\left|i^{d}-j^{d}\right| \geq C_{0} K$, we have

$$
\left|Q_{i j}\right|_{D(s-3 \sigma)} \lessdot \frac{1}{\sigma^{3 n}}\left(\frac{|\Delta \omega|+|\Delta \Omega|_{-\delta, D(s)}}{m}\left|R_{i j}\right|_{D(s-\sigma)}+\left|\Delta R_{i j}\right|_{D(s-\sigma)}\right) .
$$

Again applying Lemma 3.2 to (3.50), we have,

$$
\begin{gathered}
\left|\Delta F_{i j}\right|_{D(s-4 \sigma)} \lessdot \frac{1}{m\left|i^{d}-j^{d}\right| \sigma^{4 n}}\left(\frac{|\Delta \omega|+|\Delta \Omega|_{-\delta, D(s)}}{m}\left|R_{i j}\right|_{D(s-\sigma)}+\left|\Delta R_{i j}\right|_{D(s-\sigma)}\right), \\
\left|\left(1-\Gamma_{K}\right)\left(\tilde{\Omega}_{i j} \Delta F_{i j}\right)\right|_{D(s-4 \sigma)} \lessdot \frac{e^{-9 K \sigma / 10}}{|i-j| \sigma^{4 n}}\left(\frac{|\Delta \omega|+|\Delta \Omega|_{-\delta, D(s)}}{m}\left|R_{i j}\right|_{D(s-\sigma)}+\left|\Delta R_{i j}\right|_{D(s-\sigma)}\right) .
\end{gathered}
$$

In view of (3.52) and (3.54), applying Lemma 9.1 below again, we get the estimates of $\Delta F^{z \bar{z}}$ :

$$
\begin{aligned}
& \left\|\Delta F^{z \bar{z}}\right\|_{a, p, p, D(s-4 \sigma)},\left\|\Delta F^{z \bar{z}}\right\|_{a, q, q, D(s-4 \sigma)} \\
& \lessdot \frac{e^{8 C_{0} \gamma_{0} K s}}{\alpha \sigma^{3 n+2 \tau+1}}\left(\frac{|\Delta \omega|+|\Delta \Omega|_{-\delta, D(s)}}{\alpha}\left\|R^{z \bar{z}}\right\|_{a, q, p, D(s)}+\left\|\Delta R^{z \bar{z}}\right\|_{a, q, p, D(s)}\right) .
\end{aligned}
$$

Dividing by $|\xi-\zeta| \neq 0$ and taking the supremum over $\Pi$, we get

$$
\begin{aligned}
& \left\|F^{z \bar{z}}\right\|_{a, p, p, D(s-4 \sigma) \times \Pi}^{\text {lip }},\left\|F^{z \bar{z}}\right\|_{a, q, q, D(s-4 \sigma) \times \Pi}^{\text {lip }} \\
& \lessdot \frac{e^{8 C_{0} \gamma_{0} K s}}{\alpha \sigma^{3 n+2 \tau+1}}\left(\frac{M}{\alpha}\left\|R^{z \bar{z}}\right\|_{a, q, p, D(s) \times \Pi}+\left\|R^{z \bar{z}}\right\|_{a, q, p, D(s) \times \Pi}^{\text {lip }}\right),
\end{aligned}
$$

where $M:=|\omega|_{\Pi}^{\text {lip }}+|\Omega|_{-\delta, D(s) \times \Pi}^{\text {lip }}$. Thus, in the same way as (3.48), we get

$$
\left\|X_{\left\langle F^{z \bar{z}} z, \bar{z}\right\rangle}\right\|_{r, a, p, D(s-5 \sigma, r) \times \Pi}^{\operatorname{lip}} \lessdot \frac{e^{8 C_{0} \gamma_{0} K s}}{\alpha \sigma^{3 n+2 \tau+2}}\left(\frac{M}{\alpha}\left\|X_{R}\right\|_{r, a, q, D(s, r) \times \Pi}+\left\|X_{R}\right\|_{r, a, q, D(s, r) \times \Pi}^{\operatorname{lip}}\right) .
$$


For $\lambda \geq 0$, define

$$
\|\cdot\|_{\Pi}^{\lambda}=\|\cdot\|_{\Pi}+\lambda\|\cdot\|_{\Pi}^{\text {lip }} .
$$

The symbol ' $\lambda$ ' in $\|\cdot\|_{\Pi}^{\lambda}$ will always be used in this role and never has the meaning of exponentiation. Set $0 \leq \lambda \leq \alpha / M$. From (3.48) (3.58) we get

$$
\left\|X_{\langle F \bar{z} \bar{z}, \bar{z}\rangle}\right\|_{r, a, p, D(s-5 \sigma, r) \times \Pi}^{\lambda} \lessdot \frac{e^{8 C_{0} \gamma_{0} K s}}{\alpha \sigma^{3 n+2 \tau+2}}\left\|X_{R}\right\|_{r, a, q, D(s, r) \times \Pi}^{\lambda} \cdot
$$

Now considering the homological equations (3.13) (3.14), by a standard approach in finite dimensional KAM theory, we can easily get

$$
\begin{gathered}
\left\|X_{F^{x}}\right\|_{r, a, p, D(s-\sigma, r)}, \quad\left\|X_{\left\langle F^{y}, y\right\rangle}\right\|_{r, a, p, D(s-\sigma, r)} \lessdot \frac{1}{\alpha \sigma^{\tau+n}}\left\|X_{R}\right\|_{r, a, q, D(s, r)}, \\
\left\|X_{F^{x}}\right\|_{r, a, p, D(s-2 \sigma, r) \times \Pi}^{\text {lip }}, \quad\left\|X_{\left\langle F^{y}, y\right\rangle}\right\|_{r, a, p, D(s-2 \sigma, r) \times \Pi}^{\text {lip }} \\
\lessdot \frac{1}{\alpha \sigma^{2 \tau+2 n+1}}\left(\frac{M}{\alpha}\left\|X_{R}\right\|_{r, a, q, D(s, r) \times \Pi}+\left\|X_{R}\right\|_{r, a, q, D(s, r) \times \Pi}^{\text {iip }}\right) .
\end{gathered}
$$

From (3.60) (3.61) we get

$$
\left\|X_{F^{x}}\right\|_{r, a, p, D(s-2 \sigma, r) \times \Pi}^{\lambda}, \quad\left\|X_{\left\langle F^{y}, y\right\rangle}\right\|_{r, a, p, D(s-2 \sigma, r) \times \Pi}^{\lambda} \lessdot \frac{1}{\alpha \sigma^{2 \tau+2 n+1}}\left\|X_{R}\right\|_{r, a, q, D(s, r) \times \Pi}^{\lambda} .
$$

For the other terms of $F$, i.e. $\left\langle F^{z}, z\right\rangle,\left\langle F^{\bar{z}}, \bar{z}\right\rangle,\left\langle F^{z z} z, z\right\rangle,\left\langle F^{\bar{z} \bar{z}}, \bar{z}\right\rangle$, the same results - even better than (3.59) can be obtained. Thus, we finally get the estimate for $F$ :

$$
\left\|X_{F}\right\|_{r, a, p, D(s-5 \sigma, r) \times \Pi}^{\lambda} \lessdot \frac{e^{8 C_{0} \gamma_{0} K s}}{\alpha \sigma^{3 n+2 \tau+2}}\left\|X_{R}\right\|_{r, a, q, D(s, r) \times \Pi}^{\lambda} .
$$

\section{The New Hamiltonian}

From (3.4)-(3.12) we get the new Hamiltonian

$$
H \circ \Phi=N_{+}+P_{+},
$$

where $N_{+}=(3.4)$ and

$$
P_{+}=\hat{R}+\int_{0}^{1}\{(1-t)(\hat{N}+\hat{R})+t R, F\} \circ X_{F}^{t} d t+(P-R) \circ X_{F}^{1}
$$

where $\hat{R}=(3.7)+\cdots+(3.11):=\left\langle\hat{R}^{z}, z\right\rangle+\left\langle\hat{R}^{\bar{z}}, \bar{z}\right\rangle+\left\langle\hat{R}^{z z} z, z\right\rangle+\left\langle\hat{R}^{\bar{z} \bar{z}} \bar{z}, \bar{z}\right\rangle+\left\langle\hat{R}^{z \bar{z}} z, \bar{z}\right\rangle$. The aim of this section is to estimate the new normal form $N_{+}$and the new perturbation $P_{+}$.

\subsection{The New Normal Form}

In view of (3.4), denote $N_{+}=N+\hat{N}$ with

$$
\hat{N}=\langle\hat{\omega}, y\rangle+\sum_{j \geq 1} \hat{\Omega}_{j} z_{j} \bar{z}_{j}
$$

where

$$
\hat{\omega}:=\left[R^{y}\right],
$$




$$
\hat{\Omega}_{j}:=R_{j j}+\left\langle\partial_{x} \Omega_{j}, F^{y}\right\rangle=R_{j j}+\left\langle\partial_{x} \tilde{\Omega}_{j}, F^{y}\right\rangle .
$$

From (4.3) we easily get

$$
|\hat{\omega}|_{\Pi}^{\lambda} \lessdot\left\|X_{R}\right\|_{r, a, q, D(s, r) \times \Pi}^{\lambda} \cdot
$$

In the following, we estimate $\hat{\Omega}=\left(\hat{\Omega}_{j}: j \geq 1\right)$. In view of the second estimate of (3.60),

$$
\left|\left\langle\partial_{x} \tilde{\Omega}_{j}, F^{y}\right\rangle\right|_{D(s-\sigma)} \leq\left|\tilde{\Omega}_{j}\right|_{s, \tau+1}\left\|X_{\left\langle F^{y}, y\right\rangle}\right\|_{r, a, p, D(s-\sigma, r)} \lessdot \frac{\gamma_{0} j^{\delta}}{\sigma^{\tau+n}}\left\|X_{R}\right\|_{r, a, q, D(s, r)} .
$$

Thus, together with

$$
\left|R_{j j}\right|_{D(s-\sigma)} \leq j^{\delta}\left\|X_{R}\right\|_{r, a, q, D(s, r)},
$$

we get

$$
|\hat{\Omega}|_{-\delta, D(s-\sigma)} \lessdot \frac{1}{\sigma^{\tau+n}}\left\|X_{R}\right\|_{r, a, q, D(s, r)} .
$$

Applying $\Delta$ to $\hat{\Omega}_{j}$, we have

$$
\Delta \hat{\Omega}_{j}=\Delta R_{j j}-\left\langle\partial_{x} \Delta \tilde{\Omega}_{j}, F^{y}\right\rangle-\left\langle\partial_{x} \tilde{\Omega}_{j}, \Delta F^{y}\right\rangle .
$$

Since

$$
\begin{gathered}
\left|\Delta R_{j j}\right|_{D(s-2 \sigma)} \leq j^{\delta}\left\|\Delta X_{R}\right\|_{r, a, q, D(s, r)}, \\
\left|\left\langle\partial_{x} \Delta \tilde{\Omega}_{j}, F^{y}\right\rangle\right|_{D(s-2 \sigma)} \leq \frac{1}{\sigma}\left|\Delta \Omega_{j}\right|_{D(s-\sigma)}\left\|X_{\left\langle F^{y}, y\right\rangle}\right\|_{r, a, p, D(s-2 \sigma, r)} \lessdot \frac{|\Delta \Omega|_{-\delta, D(s)} j^{\delta}}{\alpha \sigma^{\tau+n+1}}\left\|X_{R}\right\|_{r, a, q, D(s, r)}, \\
\left|\left\langle\partial_{x} \tilde{\Omega}_{j}, \Delta F^{y}\right\rangle\right|_{D(s-2 \sigma)} \leq\left|\tilde{\Omega}_{j}\right|_{s, \tau+1}\left\|\Delta X_{\left\langle F^{y}, y\right\rangle}\right\|_{r, a, p, D(s-2 \sigma, r)} \leq \alpha \gamma_{0} j^{\delta}\left\|\Delta X_{\left\langle F^{y}, y\right\rangle}\right\|_{r, a, p, D(s-2 \sigma, r)},
\end{gathered}
$$

we get

$$
|\hat{\Omega}|_{-\delta, D(s-2 \sigma) \times \Pi}^{\operatorname{lip}} \lessdot \frac{1}{\sigma^{2 n+2 \tau+1}}\left(\frac{M}{\alpha}\left\|X_{R}\right\|_{r, a, q, D(s, r) \times \Pi}+\left\|X_{R}\right\|_{r, a, q, D(s, r) \times \Pi}^{\text {lip }}\right) .
$$

Therefore, from (4.6) (4.8) we get

$$
|\hat{\Omega}|_{-\delta, D(s-2 \sigma) \times \Pi}^{\lambda} \lessdot \frac{1}{\sigma^{2 n+2 \tau+1}}\left\|X_{R}\right\|_{r, a, q, D(s, r) \times \Pi}^{\lambda} .
$$

\subsection{The New Perturbation}

We firstly estimate the error term $\hat{R}^{z \bar{z}}$ with its matrix elements $\hat{R}_{i j}$ in (3.28). Split $\hat{R}^{z \bar{z}}$ into three parts: $\hat{R}^{z \bar{z}}=S^{1}+S^{2}+S^{3}$, such that $S^{1}, S^{2}$ have their matrix elements as follows:

$$
\begin{aligned}
& S_{i j}^{1}= \begin{cases}0, & \text { if }\left|i^{d}-j^{d}\right|<C_{0} K, \\
\left(1-\Gamma_{K}\right)\left(-\mathbf{i} \tilde{\Omega}_{i j} F_{i j}\right), & \text { if }\left|i^{d}-j^{d}\right| \geq C_{0} K,\end{cases} \\
& S_{i j}^{2}= \begin{cases}-\left(1-\Gamma_{K}\right)\left(R_{i j}\right), & \text { if } 0<\left|i^{d}-j^{d}\right|<C_{0} K, \\
0, & \text { if } i=j \text { or }\left|i^{d}-j^{d}\right| \geq C_{0} K,\end{cases}
\end{aligned}
$$

and $S^{3}$ is the cut-off of the perturbation $R^{z \bar{z}}$, that is,

$$
S^{3}=\left(1-\Gamma_{K}\right)\left(R^{z \bar{z}}-\operatorname{diag}\left(R^{z \bar{z}}\right)\right) .
$$

In view of (3.45), and using Lemma 9.1 below, we get

$$
\left\|S^{1}\right\|_{a, q, p, D(s-2 \sigma)} \lessdot \frac{e^{-9 K \sigma / 10}}{\sigma^{2 n}}\left\|R^{z \bar{z}}\right\|_{a, q, p, D(s)} .
$$


From (3.55) and

$$
\begin{aligned}
\left|\left(1-\Gamma_{K}\right)\left(\left(\Delta \tilde{\Omega}_{i j}\right) F_{i j}\right)\right|_{D(s-3 \sigma)} & \lessdot \frac{e^{-9 K \sigma / 10}}{\sigma^{2 n}}\left(i^{\delta}+j^{\delta}\right)|\Delta \Omega|_{-\delta, D(s-2 \sigma)}\left|F_{i j}\right|_{D(s-2 \sigma)} \\
& \lessdot \frac{e^{-9 K \sigma / 10}}{m|i-j| \sigma^{3 n}}|\Delta \Omega|_{-\delta, D(s-2 \sigma)}\left|R_{i j}\right|_{D(s-\sigma)},
\end{aligned}
$$

by using Lemma 9.1 below, we get

$$
\left\|S^{1}\right\|_{a, q, p, D(s-4 \sigma) \times \Pi}^{\text {lip }} \lessdot \frac{e^{-9 K \sigma / 10}}{\sigma^{5 n}}\left(\frac{M}{m}\left\|R^{z \bar{z}}\right\|_{a, q, p, D(s) \times \Pi}+\left\|R^{z \bar{z}}\right\|_{a, q, p, D(s) \times \Pi}^{\operatorname{lip}}\right) .
$$

Since

$$
\left\|S_{i j}^{2}\right\|_{D(s-2 \sigma)} \lessdot \frac{e^{-9 K \sigma / 10}}{\sigma^{n}}\left\|R_{i j}\right\|_{D(s-\sigma)},
$$

by Lemma 9.1 below, we get

$$
\begin{aligned}
\left\|S^{2}\right\|_{a, q, p, D(s-2 \sigma)} & \lessdot \frac{e^{-9 K \sigma / 10}}{\sigma^{2 n}} \max \left\{|i-j|:\left|i^{2}-j^{2}\right|<C_{0} K\right\}\left\|R^{z \bar{z}}\right\|_{a, q, p, D(s)} \\
& \lessdot \frac{C_{0} K e^{-9 K \sigma / 10}}{\sigma^{2 n}}\left\|R^{z \bar{z}}\right\|_{a, q, p, D(s)} \\
& \lessdot \frac{C_{0} e^{-4 K \sigma / 5}}{\sigma^{2 n+1}}\left\|R^{z \bar{z}}\right\|_{a, q, p, D(s)} .
\end{aligned}
$$

Again applying Lemma 9.1 to $\Delta S^{2}$, we can get

$$
\left\|S^{2}\right\|_{a, q, p, D(s-4 \sigma) \times \Pi}^{\operatorname{lip}} \lessdot \frac{C_{0} e^{-4 K \sigma / 5}}{\sigma^{2 n+1}}\left\|R^{z \bar{z}}\right\|_{a, q, p, D(s) \times \Pi}^{\text {lip }} .
$$

It is obvious that

$$
\begin{gathered}
\left\|S^{3}\right\|_{a, q, p, D(s-\sigma)} \lessdot \frac{e^{-9 K \sigma / 10}}{\sigma^{n}}\left\|R^{z \bar{z}}\right\|_{a, q, p, D(s)}, \\
\left\|S^{3}\right\|_{a, q, p, D(s-\sigma) \times \Pi} \operatorname{lip}_{\frac{e^{-9 K \sigma / 10}}{\sigma^{n}}\left\|R^{z \bar{z}}\right\|_{a, q, p, D(s) \times \Pi}^{\text {lip }}} \cdot
\end{gathered}
$$

Thus

$$
\begin{gathered}
\left\|X_{\left\langle\hat{R}^{z} \bar{z} z, \bar{z}\right\rangle}\right\|_{r, a, q, D(s-3 \sigma, r)} \lessdot \frac{\left(1+C_{0}\right) e^{-4 K \sigma / 5}}{\sigma^{2 n+2}}\left\|X_{R}\right\|_{r, a, q, D(s, r)}, \\
\left\|X_{\left\langle\hat{R}^{z} \bar{z}_{z, \bar{z}}\right\rangle}\right\|_{r, a, q, D(s-5 \sigma, r) \times \Pi}^{\operatorname{lip}} \lessdot \frac{\left(1+C_{0}\right) e^{-4 K \sigma / 5}}{\sigma^{5 n+1}}\left(\frac{M}{\alpha}\left\|X_{R}\right\|_{r, a, q, D(s, r) \times \Pi}+\left\|X_{R}\right\|_{r, a, q, D(s, r) \times \Pi}^{\operatorname{lip}}\right) .
\end{gathered}
$$

Therefore, from (4.21) (4.22) we get

$$
\left\|X_{\left\langle\hat{R}^{z \bar{z}} z, \bar{z}\right\rangle}\right\|_{r, a, q, D(s-5 \sigma, r) \times \Pi}^{\lambda} \lessdot \frac{\left(1+C_{0}\right) e^{-4 K \sigma / 5}}{\sigma^{5 n+1}}\left\|X_{R}\right\|_{r, a, q, D(s, r) \times \Pi}^{\lambda} .
$$

For the other terms of $\hat{R}$, i.e. $\left\langle\hat{R}^{z}, z\right\rangle,\left\langle\hat{R}^{\bar{z}}, \bar{z}\right\rangle,\left\langle\hat{R}^{z z} z, z\right\rangle,\left\langle\hat{R}^{z \bar{z}} \bar{z}, \bar{z}\right\rangle$, the same results - even better than (4.23) can be obtained. Thus, we finally get the estimate for the error term $\hat{R}$ :

$$
\left\|X_{\hat{R}}\right\|_{r, a, q, D(s-5 \sigma, r) \times \Pi}^{\lambda} \lessdot \frac{\left(1+C_{0}\right) e^{-4 K \sigma / 5}}{\sigma^{5 n+1}}\left\|X_{R}\right\|_{r, a, q, D(s, r) \times \Pi}^{\lambda} .
$$


Now consider the new perturbation (4.2). By setting $R(t)=(1-t)(\hat{N}+\hat{R})+t R$, we have

$$
X_{P_{+}}=X_{\hat{R}}+\int_{0}^{1}\left(X_{F}^{t}\right)^{*}\left[X_{R(t)}, X_{F}\right] d t+\left(X_{F}^{1}\right)^{*}\left(X_{P}-X_{R}\right) .
$$

We assume that

$$
\left\|X_{P}\right\|_{r, a, q, D(s, r) \times \Pi}^{\lambda} \leq \frac{\alpha \eta^{2}}{B_{\sigma}} e^{-8 C_{0} \gamma_{0} K s}
$$

for $0 \leq \lambda \leq \alpha / M$ with some $0<\eta<1 / 16$ and $0<\sigma<\min \{s, 1\}$, where $B_{\sigma}=c \sigma^{-9(n+\tau+1)}$ with $c$ being a sufficiently large constant depending only on $n, \tau$ and $|\omega|_{\Pi}$. Since $R$ is 2-order Taylor polynomial truncation in $y, z, \bar{z}$ of $P$, we can obtain

$$
\begin{gathered}
\left\|X_{R}\right\|_{r, a, q, D(s, r) \times \Pi}^{\lambda} \lessdot\left\|X_{P}\right\|_{r, a, q, D(s, r) \times \Pi}^{\lambda}, \\
\left\|X_{P}-X_{R}\right\|_{\eta r, a, q, D(s, 4 \eta r) \times \Pi}^{\lambda} \lessdot \eta\left\|X_{P}\right\|_{r, a, q, D(s, r) \times \Pi}^{\lambda} .
\end{gathered}
$$

As in lemma 19.3 of [10], we obtain

$$
\left\|D X_{F}\right\|_{r, a, p, p, D(s-6 \sigma, r) \times \Pi}^{\lambda},\left\|D X_{F}\right\|_{r, a, q, q, D(s-6 \sigma, r) \times \Pi}^{\lambda} \lessdot \frac{e^{8 C_{0} \gamma_{0} K s}}{\alpha \sigma^{3 n+2 \tau+3}}\left\|X_{R}\right\|_{r, a, q, D(s, r) \times \Pi}^{\lambda} .
$$

From (3.63), (4.5), (4.9), (4.24), (4.29) and (4.27) we get

$$
\begin{gathered}
\left\|X_{F}\right\|_{r, a, p, D(s-5 \sigma, r) \times \Pi}^{\lambda} \lessdot \frac{e^{8 C_{0} \gamma_{0} K s}}{\alpha \sigma^{3 n+2 \tau+2}}\left\|X_{P}\right\|_{r, a, q, D(s, r) \times \Pi}^{\lambda}, \\
\left\|X_{\hat{N}}\right\|_{r, a, q, D(s-2 \sigma, r) \times \Pi}^{\lambda} \lessdot \frac{1}{\sigma^{2 n+2 \tau+1}}\left\|X_{P}\right\|_{r, a, q, D(s, r) \times \Pi}^{\lambda}, \\
\left\|X_{\hat{R}}\right\|_{r, a, q, D(s-5 \sigma, r) \times \Pi}^{\lambda} \lessdot \frac{\left(1+C_{0}\right) e^{-4 K \sigma / 5}}{\sigma^{5 n+1}}\left\|X_{P}\right\|_{r, a, q, D(s, r) \times \Pi}^{\lambda}, \\
\left\|D X_{F}\right\|_{r, a, p, p, D(s-6 \sigma, r) \times \Pi}^{\lambda},\left\|D X_{F}\right\|_{r, a, q, q, D(s-6 \sigma, r) \times \Pi}^{\lambda} \lessdot \frac{e^{8 C_{0} \gamma_{0} K s}}{\alpha \sigma^{3 n+2 \tau+3}}\left\|X_{P}\right\|_{r, a, q, D(s, r) \times \Pi}^{\lambda} .
\end{gathered}
$$

Moreover, together with the smallness assumptions (4.26), by properly choosing $c$, we get

$$
\left\|X_{F}\right\|_{r, a, p, D(s-5 \sigma, r) \times \Pi}^{\lambda},\left\|D X_{F}\right\|_{r, a, p, p, D(s-6 \sigma, r) \times \Pi}^{\lambda},\left\|D X_{F}\right\|_{r, a, q, q, D(s-6 \sigma, r) \times \Pi}^{\lambda} \leq \frac{\eta^{2} \sigma}{c_{0}}
$$

with some suitable constant $c_{0} \geq 1$. Then the flow $X_{F}^{t}$ of the vector field $X_{F}$ exists on $D(s-7 \sigma, r / 2)$ for $-1 \leq t \leq 1$ and takes this domain into $D(s-6 \sigma, r)$. Similarly, it takes $D(s-8 \sigma, r / 4)$ into $D(s-7 \sigma, r / 2)$. In the same way as (20.6) in [10], we obtain

$$
\begin{gathered}
\left\|X_{F}^{t}-\mathrm{id}\right\|_{r, a, p, D(s-7 \sigma, r / 2) \times \Pi}^{\lambda} \lessdot\left\|X_{F}\right\|_{r, a, p, D(s-6 \sigma, r) \times \Pi}^{\lambda}, \\
\left\|D X_{F}^{t}-I\right\|_{r, a, p, p, D(s-8 \sigma, r / 4) \times \Pi}^{\lambda} \lessdot\left\|D X_{F}\right\|_{r, a, p, p, D(s-6 \sigma, r) \times \Pi}^{\lambda}, \\
\left\|D X_{F}^{t}-I\right\|_{r, a, q, q, D(s-8 \sigma, r / 4) \times \Pi}^{\lambda} \lessdot\left\|D X_{F}\right\|_{r, a, q, q, D(s-6 \sigma, r) \times \Pi}^{\lambda} .
\end{gathered}
$$

Also in the same way as (20.7) in [10], we obtain that for any vector field $Y$,

$$
\left\|\left(D X_{F}^{t}\right)^{*} Y\right\|_{\eta r, a, q, D(s-9 \sigma, \eta r) \times \Pi}^{\lambda} \lessdot\|Y\|_{\eta r, a, q, D(s-7 \sigma, 4 \eta r) \times \Pi}^{\lambda} \cdot
$$


From (4.27) (4.31) (4.32) and the assumption $\left(1+C_{0}\right) e^{-4 K \sigma / 5} \lessdot 1$, we get

$$
\left\|X_{R(t)}\right\|_{r, a, q, D(s-5 \sigma, r) \times \Pi}^{\lambda} \lessdot \frac{1}{\sigma^{3 n+2 \tau+1}}\left\|X_{P}\right\|_{r, a, q, D(s, r) \times \Pi}^{\lambda} .
$$

Moreover, we have

$$
\begin{aligned}
& \left\|\left[X_{R(t)}, X_{F}\right]\right\|_{r, a, q, D(s-6 \sigma, r / 2) \times \Pi}^{\lambda} \\
\lessdot & \left\|D X_{R(t)}\right\|_{r, a, q, p, D(s-6 \sigma, r / 2) \times \Pi}^{\lambda}\left\|X_{F}\right\|_{r, a, p, D(s-6 \sigma, r / 2) \times \Pi}^{\lambda} \\
& +\left\|D X_{F}\right\|_{r, a, q, q, D(s-6 \sigma, r / 2) \times \Pi}^{\lambda}\left\|X_{R(t)}\right\|_{r, a, q, D(s-6 \sigma, r / 2) \times \Pi}^{\lambda} \\
\lessdot & \frac{e^{8 C_{0} \gamma_{0} K s}}{\alpha \sigma^{6 n+4 \tau+4}}\left(\left\|X_{P}\right\|_{r, a, q, D(s, r) \times \Pi}^{\lambda}\right)^{2} .
\end{aligned}
$$

Hence, also

$$
\left\|\left[X_{R(t)}, X_{F}\right]\right\|_{\eta r, a, q, D(s-6 \sigma, r / 2) \times \Pi}^{\lambda} \lessdot \frac{e^{8 C_{0} \gamma_{0} K s}}{\alpha \eta^{2} \sigma^{6 n+4 \tau+4}}\left(\left\|X_{P}\right\|_{r, a, q, D(s, r) \times \Pi}^{\lambda}\right)^{2} .
$$

Together with the estimates of $\hat{R}$ in (4.32) and $X_{P}-X_{R}$ in (4.28), we finally arrive at the estimate

$$
\begin{aligned}
& \left\|X_{P_{+}}\right\|_{\eta r, a, q, D(s-9 \sigma, \eta r) \times \Pi}^{\lambda} \\
\leq & \frac{1}{3}\left(\frac{B_{\sigma} e^{8 C_{0} \gamma_{0} K s}}{\alpha \eta^{2}}\left\|X_{P}\right\|_{r, a, q, D(s, r) \times \Pi}^{\lambda}+\frac{B_{\sigma} e^{-4 K \sigma / 5}}{\alpha \eta^{2}}+\eta\right)\left\|X_{P}\right\|_{r, a, q, D(s, r) \times \Pi}^{\lambda} .
\end{aligned}
$$

This is the bound for the new perturbation.

\section{Iteration and Convergence}

Set $\beta^{\prime}=\min \left\{\frac{\beta}{1+\beta}, \frac{1}{4}\right\}$ and $\kappa=\frac{4}{3}-\frac{\beta^{\prime}}{3}$. Now we give the precise set-up of iteration parameters. Let $v \geq 0$ be the $v$-th KAM step.

$\alpha_{v}=\frac{\alpha_{0}}{10}\left(9+2^{-v}\right)$, which is used to dominate the measure of removed parameters,

$m_{v}=\frac{m_{0}}{10}\left(9+2^{-v}\right)$, which is used for describing the growth of external frequencies,

$E_{v}=\frac{E_{0}}{9}\left(10-2^{-v}\right)$, which is used to dominate the norm of internal frequencies,

$M_{1, v}=\frac{M_{1,0}}{9}\left(10-2^{-v}\right), \quad M_{2, v}=\frac{M_{2,0}}{9}\left(10-2^{-v}\right), \quad M_{v}=M_{1, v}+M_{2, v}$, which are used to dominate the Lipschitz semi-norm of frequencies,

$L_{v}=\frac{L_{0}}{9}\left(10-2^{-v}\right)$, which is used to dominate the inverse Lipschitz semi-norm of internal frequencies,

$J_{0}=\gamma_{0}^{-\frac{1}{\tau+1}}, \quad J_{v}=J_{0}^{\kappa^{v}}$, which is used for estimate of measure,

$s_{v}=s_{0} / 2^{v}$, which dominates the width of the angle variable $x$,

$\sigma_{v}=s_{v} / 20$, which serves as a bridge from $s_{V}$ to $s_{v+1}$,

$B_{v}=B_{\sigma_{v}}:=c \sigma_{v}^{-9(n+\tau+1)}$, here $c$ is a large constant only depending on $n, \tau$ and $E_{0}$, 
$\varepsilon_{v}=\left(\varepsilon_{0} \prod_{\mu=0}^{v-1}\left(\frac{B_{\mu}}{\alpha_{\mu}}\right)^{\frac{1}{3 \kappa^{\mu+1}}}\right)^{\kappa^{\nu}}$, which dominates the size of the perturbation $P_{v}$ in $v$-the KAM iteration,

$K_{v}=5\left|\ln \varepsilon_{v}\right| /\left(4 \sigma_{v}\right)$, which is the length of the truncation of Fourier series,

$\eta_{v}^{3}=\varepsilon_{v}^{1-\beta^{\prime}} \alpha_{v}^{-1} B_{v}, \quad r_{v+1}=\eta_{v} r_{v}, \quad D_{v}=D\left(s_{v}, r_{v}\right), \quad \lambda_{v}=\frac{\alpha_{v}}{M_{v}}$.

\subsection{Iterative Lemma}

Lemma 5.1. Suppose that

$$
\varepsilon_{0} \leq\left(\frac{\alpha_{0} \gamma_{0}}{80}\right)^{\frac{1}{1-\beta^{\prime}}} \prod_{\mu=0}^{\infty} B_{\mu}^{-\frac{1}{3 \kappa^{\mu+1}}}, \quad \alpha_{0} \leq \frac{m_{0}}{10} .
$$

Suppose $H_{v}=N_{v}+P_{v}$ is regular on $D_{v} \times \Pi_{v}$, where $N_{v}$ is a generalized normal form with coefficients satisfying

$$
\begin{gathered}
\left|\left\langle k, \omega_{v}(\xi)\right\rangle+\left\langle l, \bar{\Omega}_{v}(\xi)\right\rangle\right| \geq \alpha_{v} \frac{\langle l\rangle_{d}}{\langle k\rangle}, \quad k \neq 0, \quad|l| \leq 2, \\
\left|\left\langle l, \bar{\Omega}_{v}(\xi)\right\rangle\right| \geq m_{v}\langle l\rangle_{d}, \quad 0<|l| \leq 2, \\
\left|\tilde{\Omega}_{v, j}\right|_{s_{v}, \tau+1} \leq\left(\alpha_{0}-\alpha_{v}\right) \gamma_{0} j^{\delta}, \quad j \geq 1, \\
\left|\omega_{v}\right|_{\Pi_{v}} \leq E_{v}, \quad\left|\omega_{v}\right|_{\Pi_{v}}^{\operatorname{lip}_{\nu}} \leq M_{1, v}, \quad\left|\omega_{v}^{-1}\right|_{\omega_{v}\left(\Pi_{v}\right)}^{\operatorname{lip}} \leq L_{v}, \\
\left|\Omega_{v}\right|_{-\delta, D_{v} \times \Pi_{v}}^{\operatorname{lip}} \leq M_{2, v}
\end{gathered}
$$

on $\Pi_{v}$, and $P_{v}$ satisfies

$$
\left\|X_{P_{v}}\right\|_{r_{v}, a, q, D_{v} \times \Pi_{v}}^{\lambda_{v}} \leq \varepsilon_{v}
$$

Then there exists a Lipschitz family of real analytic symplectic coordinate transformations $\Phi_{v+1}$ : $D_{v+1} \times \Pi_{v} \rightarrow D_{v}$ satisfying

$$
\left\|\Phi_{v+1}-\mathrm{id}\right\|_{r_{v}, a, p, D_{v+1} \times \Pi_{v}}^{\lambda_{v}},\left\|D \Phi_{v+1}-I\right\|_{r_{v}, a, p, p, D_{v+1} \times \Pi_{v}}^{\lambda_{v}},\left\|D \Phi_{v+1}-I\right\|_{r_{v}, a, q, q, D_{v+1} \times \Pi_{v}}^{\lambda_{\lambda}} \leq \frac{B_{v}}{\alpha_{v}} \varepsilon_{v}^{1-\beta^{\prime}},
$$

and a closed subset

$$
\Pi_{v+1}=\Pi_{v} \backslash \bigcup_{|k|>J_{v},|l| \leq 2} \mathfrak{R}_{k l}^{v+1}\left(\alpha_{v+1}\right)
$$

where

$$
\mathfrak{R}_{k l}^{v+1}(\alpha)=\left\{\xi \in \Pi_{v}:\left|\left\langle k, \omega_{v+1}(\xi)\right\rangle+\left\langle l, \bar{\Omega}_{v+1}(\xi)\right\rangle\right|\left\langle\alpha \frac{\langle l\rangle_{d}}{\langle k\rangle^{\tau}}\right\},\right.
$$

such that for $H_{v+1}=H_{v} \circ \Phi_{v+1}=N_{v+1}+P_{v+1}$, the estimate

$$
\left|\omega_{v+1}-\omega_{v}\right|_{\Pi_{v}}^{\lambda_{v}}, \quad\left|\Omega_{v+1}-\Omega_{v}\right|_{-\delta, D_{v+1} \times \Pi_{v}}^{\lambda_{v}} \leq B_{v} \varepsilon_{v}
$$

holds and the same assumptions as above are satisfied with ' $v+1$ ' in place of ' $v$ '.

Proof. Setting $C_{0, v}=2 E_{v} / m_{v}$, then it's obvious $C_{0, v} \leq 4 C_{0,0}$. Thus we have

$$
e^{8 C_{0, v} \gamma_{0} K_{v} s_{v}} \leq \varepsilon_{v}^{-\beta^{\prime}}
$$


by $K_{v} s_{V}=20 K_{v} \sigma_{v}=25\left|\ln \varepsilon_{v}\right|$ and choosing $\gamma_{0}$ small enough such that $800 C_{0,0} \gamma_{0} \leq \beta^{\prime}$. In view of the definition of $\eta_{v}$, namely $\eta_{v}^{3}=\varepsilon_{v}^{1-\beta^{\prime}} \alpha_{v}^{-1} B_{v}$, the smallness condition (4.26), namely $\varepsilon_{v} \leq$ $\frac{\alpha_{v} \eta_{v}^{2}}{B_{v}} e^{-8 C_{0, v} \gamma_{0} K_{v} s_{v}}$, is satisfied if

$$
\varepsilon_{v}^{1-\beta^{\prime}} \leq \frac{\alpha_{v}}{B_{v}}
$$

To verify the last inequality we argue as follows. As $B_{v}$ and $\alpha_{v}^{-1}$ are increasing with $v$,

$$
\left(\frac{B_{v}}{\alpha_{v}}\right)^{\frac{1}{1-\beta^{\prime}}}=\left(\frac{B_{v}}{\alpha_{v}}\right)^{\frac{1}{3(\kappa-1)}}=\left(\prod_{\mu=v}^{\infty}\left(\frac{B_{v}}{\alpha_{v}}\right)^{\frac{1}{3 \kappa^{\mu+1}}}\right)^{\kappa^{v}} \leq\left(\prod_{\mu=v}^{\infty}\left(\frac{B_{\mu}}{\alpha_{\mu}}\right)^{\frac{1}{3 \kappa^{\mu+1}}}\right)^{\kappa^{v}} .
$$

By the definition of $\varepsilon_{v}$ above, the bound $\alpha_{v} \geq 9 \alpha_{0} / 10$ and the smallness condition on $\varepsilon_{0}$ in (5.1),

$$
\varepsilon_{v}^{1-\beta^{\prime}} \frac{B_{v}}{\alpha_{v}} \leq\left(\varepsilon_{0} \prod_{\mu=0}^{\infty}\left(\frac{B_{\mu}}{\alpha_{\mu}}\right)^{\frac{1}{3 \kappa^{\mu+1}}}\right)^{\kappa^{v}\left(1-\beta^{\prime}\right)} \leq\left(\frac{\gamma_{0}}{72}\right)^{\kappa^{v}} \leq 1 .
$$

So the smallness condition (4.26) is satisfied for each $v \geq 0$. In particular, noticing $\kappa \geq 5 / 4$, we have

$$
\varepsilon_{v}^{1-\beta^{\prime}} \frac{B_{v}}{\alpha_{v}} \leq \frac{\gamma_{0}}{2^{v+6}}
$$

Now there exists a coordinate transformation $\Phi_{v+1}: D_{v+1} \times \Pi_{v} \rightarrow D_{v}$ taking $H_{v}$ into $H_{v+1}$. Moreover, (5.8) is obtained by (4.30) (4.33) (4.35)-(4.37), and (5.11) is obtained by (4.31). More explicitly, (5.11) is written as

$$
\begin{gathered}
\left|\omega_{v+1}-\omega_{v}\right|_{\Pi_{v}}, \quad\left|\Omega_{v+1}-\Omega_{v}\right|_{-\delta, D_{v+1} \times \Pi_{v}} \leq B_{v} \varepsilon_{v}, \\
\left|\omega_{v+1}-\omega_{v}\right|_{\Pi_{v}}^{\operatorname{lip}}, \quad\left|\Omega_{v+1}-\Omega_{v}\right|_{-\delta, D_{v+1} \times \Pi_{v}}^{\operatorname{lip}} \leq \frac{M_{v}}{\alpha_{v}} B_{v} \varepsilon_{v} .
\end{gathered}
$$

In view of (5.16)-(5.18), by choosing $\gamma_{0}$ properly small, (5.3)-(5.6) are satisfied with ' $v+1$ ' in place of ' $v$ '. As to the diophantine conditions, in view of the definition of $\Pi_{v+1}$ in (5.9), only the case $\langle k\rangle \leq J_{v}$ remains to verify. In this case, by the definition of $J_{v}$, we have

$$
B_{v} \varepsilon_{v} \leq \frac{\alpha_{v} \gamma_{0}^{\kappa^{v}}}{2^{v+6}} \leq \frac{\alpha_{v}-\alpha_{v+1}}{3 J_{v}^{\tau+1}}
$$

Hence, for $k \neq 0$ and $\langle k\rangle \leq J_{v}$,

$$
\begin{aligned}
\left|\left\langle k, \omega_{v+1}-\omega_{v}\right\rangle+\left\langle l, \bar{\Omega}_{v+1}-\bar{\Omega}_{v}\right\rangle\right| & \leq|k|\left|\omega_{v+1}-\omega_{v}\right|+2\langle l\rangle_{d}\left|\bar{\Omega}_{v+1}-\bar{\Omega}_{v}\right|_{-\delta} \\
& \leq 3|k|\langle l\rangle_{d} B_{v} \varepsilon_{v} \\
& \leq\left(\alpha_{v}-\alpha_{v+1}\right) \frac{|k|\langle l\rangle_{d}}{J_{v}^{\tau+1}} \\
& \leq\left(\alpha_{v}-\alpha_{v+1}\right) \frac{\langle l\rangle_{d}}{\langle k\rangle^{\tau}}
\end{aligned}
$$

on $D_{v+1} \times \Pi_{v}$. This completes the proof of (5.2) with ' $v+1$ ' in place of ' $v$ '. On the other hand, from (4.42) we get

$$
\begin{aligned}
\left\|X_{P_{v+1}}\right\|_{r_{v+1}, a, q, D_{v+1} \times \Pi_{v}}^{\lambda_{v+1}} & \leq \frac{1}{3}\left(\frac{B_{v} e^{8 C_{0, v} \gamma_{0} K_{v} s_{v}}}{\alpha_{v} \eta_{v}^{2}} \varepsilon_{v}+\frac{B_{v} e^{-4 K_{v} \sigma_{v} / 5}}{\alpha_{v} \eta_{v}^{2}}+\eta_{v}\right) \varepsilon_{v} \\
& \leq \frac{1}{3}\left(\frac{B_{v}}{\alpha_{v} \eta_{v}^{2}} \varepsilon_{v}^{1-\beta^{\prime}}+\frac{B_{v}}{\alpha_{v} \eta_{v}^{2}} \varepsilon_{v}^{1-\beta^{\prime}}+\eta_{v}\right) \varepsilon_{v} \\
& =\left(\frac{B_{v}}{\alpha_{v}}\right)^{\frac{1}{3}} \varepsilon_{v}^{\kappa} \\
& =\varepsilon_{v+1} .
\end{aligned}
$$


This completes the proof of iterative lemma.

\subsection{Convergence}

We are now in a position to prove the KAM theorem. To apply iterative lemma with $v=0$, set

$$
N_{0}=N, \quad P_{0}=P, \quad s_{0}=s, \quad r_{0}=r
$$

and similarly $E_{0}=E, L_{0}=L, M_{1,0}=M_{1}, M_{2,0}=M_{2}, m_{0}=m, \alpha_{0}=\alpha$ and $\lambda_{0}=\lambda=\alpha / M$. Define $\gamma$ in the KAM theorem by setting

$$
\gamma=\gamma_{0} \gamma_{s}, \quad \gamma_{s}=\frac{1}{80}\left(\prod_{\mu=0}^{\infty} B_{\mu}^{-\frac{1}{3 k^{\mu+1}}}\right)^{1-\beta^{\prime}},
$$

where $\gamma_{0}$ is the same parameter as before and $\gamma_{s}$ only depends on $n, \tau, E, s, \beta$. The smallness condition (5.1) of the iterative lemma is then satisfied by the assumption of the KAM theorem:

$$
\varepsilon_{0}:=\left\|X_{P_{0}}\right\|_{r_{0}, a, q, D_{0} \times \Pi_{0}}^{\lambda_{0}} \leq(\alpha \gamma)^{1+\beta} \leq\left(\alpha_{0} \gamma_{0} \gamma_{s}\right)^{\frac{1}{1-\beta^{\prime}}} .
$$

The small divisor conditions (5.2) are satisfied by setting

$$
\Pi_{0}=\Pi \backslash \bigcup_{(k, l) \neq(0,0),|l| \leq 2} \mathfrak{R}_{k l}^{0}\left(\alpha_{0}\right),
$$

and the other conditions (5.3)-(5.6) about the unperturbed frequencies are obviously true.

Hence, the iterative lemma applies, and we obtain a decreasing sequence of domains $D_{v} \times \Pi_{v}$ and a sequence of transformations

$$
\Phi^{v}=\Phi_{1} \circ \cdots \circ \Phi_{v}: \quad D_{v} \times \Pi_{v-1} \rightarrow D_{0},
$$

such that $H \circ \Phi^{v}=N_{v}+P_{v}$ for $v \geq 1$. Moreover, the estimates (5.8) and (5.11) hold.

Shorten $\|\cdot\|_{r, a, p}$ as $\|\cdot\|_{r}$ and consider the operator norm

$$
\|L\|_{r, \tilde{r}}=\sup _{W \neq 0} \frac{\|L W\|_{r}}{\|W\|_{\tilde{r}}} .
$$

For $r \geq \tilde{r}$, these norms satisfy $\|A B\|_{r, \tilde{r}} \leq\|A\|_{r, r}\|B\|_{\tilde{r}, \tilde{r}}$ since $\|W\|_{r} \leq\|W\|_{\tilde{r}}$. For $v \geq 1$, by the chain rule, using (5.8) (5.16), we get

$$
\begin{gathered}
\left\|D \Phi^{v}\right\|_{r_{0}, r_{v}, D_{v} \times \Pi_{v-1}} \leq \prod_{\mu=1}^{v}\left\|D \Phi_{\mu}\right\|_{r_{\mu}, r_{\mu}, D_{\mu} \times \Pi_{\mu-1}} \leq \prod_{\mu=1}^{\infty}\left(1+\frac{1}{2^{\mu+6}}\right) \leq 2, \\
\left\|D \Phi^{v}\right\|_{r_{0}, r_{v}, D_{v} \times \Pi_{v-1}}^{\operatorname{lip}_{0}} \leq \sum_{\mu=1}^{v}\left\|D \Phi_{\mu}\right\|_{r_{\mu}, r_{\mu}, D_{\mu} \times \Pi_{\mu-1}} \prod_{1 \leq \rho \leq v, \rho \neq \mu}\left\|D \Phi_{\rho}\right\|_{r_{\rho}, r_{\rho}, D_{\rho} \times \Pi_{\rho-1}} \\
\leq 2 \sum_{\mu=1}^{v}\left\|D \Phi_{\mu}-I\right\|_{r_{\mu}, r_{\mu}, D_{\mu} \times \Pi_{\mu-1}}^{\operatorname{lip}_{1}} \leq 2 \prod_{\mu=1}^{\infty} \frac{M_{\mu}}{\alpha_{\mu}} \frac{1}{2^{\mu+6}} \leq \frac{M_{0}}{\alpha_{0}} .
\end{gathered}
$$

Thus, with the mean value theorem we obtain

$$
\begin{aligned}
& \left\|\Phi^{v+1}-\Phi^{v}\right\|_{r_{0}, D_{v+1} \times \Pi_{v}} \\
& \leq\left\|D \Phi^{v}\right\|_{r_{0}, r_{v}, D_{v} \times \Pi_{v-1}}\left\|\Phi_{v+1}-\mathrm{id}\right\|_{r_{v}, D_{v+1} \times \Pi_{v}} \leq 2\left\|\Phi_{v+1}-\mathrm{id}\right\|_{r_{v}, D_{v+1} \times \Pi_{v}},
\end{aligned}
$$




$$
\begin{aligned}
& \left\|\Phi^{v+1}-\Phi^{v}\right\|_{r_{0}, D_{v+1} \times \Pi_{v}}^{\text {lip }} \\
& \leq\left\|D \Phi^{v}\right\|_{r_{0}, r_{v}, D_{v} \times \Pi_{v-1}}^{\text {inp }}\left\|\Phi_{v+1}-\mathrm{id}\right\|_{r_{v}, D_{v+1} \times \Pi_{v}}+\left\|D \Phi^{v}\right\|_{r_{0}, r_{v}, D_{v} \times \Pi_{v-1}}\left\|\Phi_{v+1}-\mathrm{id}\right\|_{r_{v}, D_{v+1} \times \Pi_{v}}^{\operatorname{lip}} \\
& \leq \frac{M_{0}}{\alpha_{0}}\left\|\Phi_{v+1}-\mathrm{id}\right\|_{r_{v}, D_{v+1} \times \Pi_{v}}+2\left\|\Phi_{v+1}-\mathrm{id}\right\|_{r_{v}, D_{v+1} \times \Pi_{v}}^{\operatorname{lip}} .
\end{aligned}
$$

It follows that

$$
\left\|\Phi^{v+1}-\Phi^{v}\right\|_{r_{0}, D_{v+1} \times \Pi_{v}}^{\lambda_{0}} \leq 3\left\|\Phi_{v+1}-\mathrm{id}\right\|_{r_{v}, D_{v+1} \times \Pi_{v}}^{\lambda_{v}} .
$$

From (5.29) (5.8), we get

$$
\left\|\Phi^{v+1}-\Phi^{v}\right\|_{r_{0}, D_{v+1} \times \Pi_{v}}^{\lambda_{0}} \leq 3 \frac{B_{v}}{\alpha_{v}} \varepsilon_{v}^{1-\beta^{\prime}} .
$$

For every non-negative integer multi-index $k=\left(k_{1}, \ldots, k_{n}\right)$, by Cauchy's estimate we have

$$
\left\|\partial_{x}^{k}\left(\Phi^{v+1}-\Phi^{v}\right)\right\|_{r_{0}, D_{v+2} \times \Pi_{v}}^{\lambda_{0}} \leq 3 \frac{B_{v}}{\alpha_{v}} \varepsilon_{v}^{1-\beta^{\prime}} \frac{k_{1} ! \cdots k_{n} !}{\left(\frac{s_{0}}{2^{v+2}}\right)^{|k|}},
$$

the right side of which super-exponentially decay with $v$. This shows that $\Phi^{v}$ converge uniformly on $D_{*} \times \Pi_{\alpha}$, where $D_{*}=\mathbb{T}^{n} \times\{0\} \times\{0\} \times\{0\}$ and $\Pi_{\alpha}=\bigcap_{v \geq 0} \Pi_{v}$, to a Lipschitz continuous family of smooth torus embeddings

$$
\Phi: \mathbb{T}^{n} \times \Pi_{\alpha} \rightarrow \mathscr{P} a, p,
$$

for which the estimate (1.9) holds. Similarly, the frequencies $\omega_{v}$ converge uniformly on $\Pi_{\alpha}$ to a Lipschitz continuous limit $\omega_{*}$, and the frequencies $\Omega_{v}$ converge uniformly on $D_{*} \times \Pi_{\alpha}$ to a regular limit $\Omega_{*}$, with estimate (1.10) holding. Moreover, $X_{H} \circ \Phi=D \Phi \cdot X_{N_{*}}$ on $D_{*}$ for each $\xi \in \Pi_{\alpha}$, where $N_{*}$ is the generalized normal form with frequencies $\omega_{*}$ and $\Omega_{*}$. Thus, the embedded tori are invariant under the perturbed Hamiltonian flow, and the flow on them is linear. Now it only remains to prove the claim about the set $\Pi \backslash \Pi_{\alpha}$, which is the subject of the next section.

\section{Measure Estimate}

\subsection{Proof of (1.8)}

We know

$$
\Pi \backslash \Pi_{\alpha}=\bigcup_{v \geq 0} \bigcup_{|k|>J_{v-1},|l| \leq 2} \Re_{k l}^{v},
$$

where $J_{-1}=0, J_{v}=\gamma_{0}^{-\kappa^{v} /(\tau+1)}$ and

$$
\mathfrak{R}_{k l}^{v}=\left\{\xi \in \Pi_{v-1}:\left|\left\langle k, \omega_{v}(\xi)\right\rangle+\left\langle l, \bar{\Omega}_{v}(\xi)\right\rangle\right|<\alpha_{v} \frac{\langle l\rangle_{d}}{\langle k\rangle^{\tau}}\right\}
$$

with $\Pi_{-1}=\Pi$. Here, $\omega_{v}$ and $\bar{\Omega}_{v}$ are defined and Lipschitz continuous on $\Pi_{v-1}$, and $\omega_{0}=\omega, \bar{\Omega}_{0}=\Omega$ are the frequencies of the unperturbed system.

Lemma 6.1. If $\gamma_{0}$ is sufficiently small and $\tau \geq n+1+2 /(d-1)$, then

$$
\left|\Pi \backslash \Pi_{\alpha}\right| \leq c \alpha,
$$

where $c>0$ is a constant depends on $n, d, E, L$ and $m$. 
Proof. We only need to give the proof of the most difficult case that $l$ has two non-zero components of opposite sign. In this case, rewriting $\mathfrak{R}_{k l}^{v}$ as

$$
\mathfrak{R}_{k i j}^{v}=\left\{\xi \in \Pi_{v-1}:\left|\left\langle k, \omega_{v}(\xi)\right\rangle+\bar{\Omega}_{v, i}(\xi)-\bar{\Omega}_{v, j}(\xi)\right|<\alpha_{v} \frac{\left|i^{d}-j^{d}\right|}{\langle k\rangle^{\tau}}\right\}, i \neq j,
$$

we only need to estimate the measure of

$$
\Xi_{\alpha}:=\bigcup_{v \geq 0} \bigcup_{|k|>J_{v-1}, i \neq j} \Re_{k i j}^{v} .
$$

To estimate the measure of $\bigcup_{|k|>J_{v-1}, i \neq j} \Re_{k i j}^{v}$, we introduce the perturbed frequencies $\zeta=\omega_{v}(\xi)$ as parameters over the domain $Z:=\omega_{v}\left(\Pi_{v}\right)$ and consider the resonance zones $\dot{\mathfrak{R}}_{k i j}^{v}=\omega_{v}\left(\mathfrak{R}_{k i j}^{v}\right)$ in $Z$. Regarding $\bar{\Omega}_{v}$ as function of $\zeta$, then from the iterative lemma above, we know

$$
\begin{gathered}
|\zeta| \leq E_{v} \leq \frac{10}{9} E_{0}, \quad|\xi|_{Z}^{\text {lip }} \leq L_{v} \leq \frac{10}{9} L_{0}, \\
\left|\bar{\Omega}_{v, i}-\bar{\Omega}_{v, j}\right| Z \geq m_{v}\left|i^{d}-j^{d}\right| \geq \frac{9}{10} m_{0}\left|i^{d}-j^{d}\right|, \\
\left|\bar{\Omega}_{v, j}\right|_{Z}^{\text {lip }} \leq L_{v} M_{2, v} j^{\delta} \leq\left(\frac{10}{9}\right)^{2} L_{0} M_{2,0} j^{\delta},
\end{gathered}
$$

where $E_{0}, L_{0}, m_{0}, M_{2,0}$ are just $E, L, m, M_{2}$ in theorem 1.1.

Now we consider a fixed $\dot{R}_{k i j}^{v}$.

If $|k|<\frac{9 m_{v}}{10 E_{v}}\left|i^{d}-j^{d}\right|$, we get $|\langle k, \zeta\rangle|<\frac{9 m_{v}}{10}\left|i^{d}-j^{d}\right|$. In view of (6.7) and $\alpha_{v} \leq \frac{m_{v}}{10}$, we know $\dot{\mathfrak{R}}_{k i j}^{v}$ is empty.

If $|k| \geq \frac{9 m_{v}}{10 E_{v}}\left|i^{d}-j^{d}\right|$, we have $|k| \geq \frac{1}{2}\left(\frac{9}{10}\right)^{3} \frac{m}{E}\left(i^{\delta}+j^{\delta}\right)$. Fix $w_{1} \in\{-1,1\}^{n}$ such that $|k|=k \cdot w_{1}$ and write $\zeta=a w_{1}+w_{2}$ with $w_{1} \perp w_{2}$. As a function of $a$, for $t>s$,

$$
\begin{gathered}
\left.\langle k, \zeta\rangle\right|_{s} ^{t}=|k|(t-s), \\
\left.\left(\left|\bar{\Omega}_{v, i}-\bar{\Omega}_{v, j}\right|\right)\right|_{s} ^{t} \leq\left(\frac{10}{9}\right)^{2} L M_{2}\left(i^{\delta}+j^{\delta}\right)(t-s) .
\end{gathered}
$$

Thus

$$
\begin{aligned}
\left.\left(\langle k, \zeta\rangle+\bar{\Omega}_{v, i}-\bar{\Omega}_{v, j}\right)\right|_{s} ^{t} & \geq|k|(t-s)\left(1-\left(\frac{9}{10}\right)^{2} L M_{2}\left(i^{\delta}+j^{\delta}\right) /|k|\right) \\
& \geq|k|(t-s)\left(1-2\left(\frac{10}{9}\right)^{5} \frac{E L M_{2}}{m}\right) \\
& \geq \frac{1}{10}|k|(t-s),
\end{aligned}
$$

by using the assumption $4 E L M_{2} \leq m$ in theorem 1.1 and the fact $(9 / 10)^{6}>1 / 2$. Therefore, we get

$$
\left|\dot{\mathfrak{R}}_{k i j}^{v}\right| \leq 10(\operatorname{diam} Z)^{n-1} \alpha_{v} \frac{\left|i^{d}-j^{d}\right|}{\langle k\rangle^{\tau+1}} \leq 10\left(\frac{20}{9} E\right)^{n-1} \alpha \frac{\left(\frac{10}{9}\right)^{3} \frac{E}{m}}{\langle k\rangle^{\tau}} .
$$

Going back to the original parameter domain $\Pi_{v}$ by the inverse frequency map $\omega^{-1}$, we get

$$
\left|\Re_{k i j}^{v}\right| \leq c_{1} \frac{\alpha}{\langle k\rangle^{\tau}},
$$


where $c_{1}=5(10 / 9)^{2 n+2}(2 E L)^{n} / m$. Consequently, we have that for any $|k|>J_{v-1}$,

$$
\left|\bigcup_{i \neq j} \Re_{k i j}^{v}\right| \leq \bigcup_{\left|i^{d}-j^{d}\right| \leq(10 / 9)^{3}(E / m)|k|}\left|\Re_{k i j}^{v}\right| \leq c_{2} \frac{\alpha}{\langle k\rangle^{\tau-\frac{2}{d-1}}},
$$

where $c_{2}=c_{1}\left(2(10 / 9)^{3}(E / m)\right)^{2 /(d-1)}$. Moreover, since $\tau \geq n+1+2 /(d-1)$, we have

$$
\left|\bigcup_{|k|>J_{v-1}, i \neq j} \Re_{k i j}^{v}\right| \leq c_{2} c_{3} \frac{\alpha}{1+J_{v-1}}
$$

where $c_{3}>0$ depends only on $n$. The sum of the latter inequality over all $v$ converges, and we finally obtain the estimate of lemma 6.1 .

\subsection{Proof of Theorem 1.2}

Since the proof of the case $\delta=\tilde{d}<d-1$ can be found in [7] or [10], we assume $\delta=\tilde{d}=d-1$ in the following.

From the assumption (D*) we know $\bar{\Omega}_{0}=\Omega^{1}+\Omega^{2}$ with $\left|\Omega^{1}\right|_{-\delta_{0}, \Pi}^{\text {lip }} \leq M_{3}$ and $\left|\Omega^{2}\right|_{-\delta, \Pi}^{\text {lip }} \leq M_{4}$. Thus in $v$-th KAM step, setting $\bar{\Omega}_{v}=\Omega^{1}+\Omega_{v}^{2}$, we know $\Omega_{0}^{2}=\Omega^{2}$ and

$$
\left|\Omega_{v}^{2}\right|_{-\delta, \Pi}^{\operatorname{lip}} \leq M_{4, v}
$$

where the iteration parameter $M_{4, v}=\frac{M_{4}}{9}\left(10-2^{-v}\right)$.

In the following we consider the excluded set of parameters under the assumption $\left(D^{*}\right)$ instead of (D). Our aim is to verify the conclusion $\left(1^{*}\right)$ in Theorem 1.2.

In the same way as [10], we write $\Pi \backslash \Pi_{\alpha}=\Xi_{\alpha}^{1}+\Xi_{\alpha}^{2}$, where

$$
\begin{gathered}
\Xi_{\alpha}^{1}=\bigcup_{0<|k| \leq J_{0},|l| \leq 2} \Re_{k l}^{0}, \\
\Xi_{\alpha}^{2}=\bigcup_{v \geq 0} \bigcup_{|k|>\max \left(J_{0}, J_{v-1}\right),|l| \leq 2} \mathfrak{R}_{k l}^{v} .
\end{gathered}
$$

Since $|k| \leq J_{0}$ and for each $k$ there are only finitely many $l$ for which $\mathfrak{R}_{k l}^{0}$ is not empty, the set $\Xi_{\alpha}^{1}$ is a finite union of resonance zones. For each of its members we know that $\left|\mathfrak{R}_{k l}^{0}\right| \rightarrow 0$ as $\alpha \rightarrow 0$ for $l \neq 0$ by the first part of assumption ( $\mathrm{D}^{*}$ ), and for $l=0$ by elementary volume estimates. Thus $\left|\Xi_{\alpha}^{1}\right| \rightarrow 0$ as $\alpha \rightarrow 0$. In the remainder of this section we estimate the measure of $\Xi_{\alpha}^{2}$.

Lemma 6.2. If $\gamma_{0}$ is sufficiently small and $\tau \geq n+1+2 /(d-1)$, then

$$
\left|\Xi_{\alpha}^{2}\right| \leq c \alpha
$$

where $c>0$ is a constant depends on $n, d, E, L$ and $m$.

Proof. As in Lemma 6.1, we only need to give the proof of the most difficult case that $l$ has two nonzero components of opposite sign. Seeing (6.4) for the definition of $\Re_{k i j}^{v}$, we only need to estimate the measure of

$$
\bigcup_{v \geq 0} \bigcup_{|k|>\max \left(J_{0}, J_{v-1}\right), i \neq j} \Re_{k i j}^{v} .
$$


For the measure of $\bigcup_{|k|>\max \left(J_{0}, J_{v-1}\right), i \neq j} \Re_{k i j}^{v}$, we introduce the perturbed frequencies $\zeta=\omega_{v}(\xi)$ as parameters over the domain $Z=\omega_{v}\left(\Pi_{v}\right)$ and consider the resonance zones $\dot{\mathfrak{R}}_{k i j}^{v}=\omega_{v}\left(\mathfrak{R}_{k i j}^{v}\right)$ in $Z$. The estimate (6.6) (6.7) still hold true. Moreover, for $\bar{\Omega}_{v, j}=\Omega_{j}^{1}+\Omega_{v, j}^{2}$, we have the estimate

$$
\begin{gathered}
\left|\Omega_{j}^{1}\right|_{Z}^{\operatorname{lip}} \leq L_{v} M_{3} j^{\delta_{0}} \leq \frac{10}{9} L M_{3} j^{\delta_{0}}, \\
\left|\Omega_{v, j}^{2}\right|_{Z}^{\operatorname{lip}} \leq L_{v} M_{4, v} j^{\delta} \leq\left(\frac{10}{9}\right)^{2} L M_{4} j^{\delta} .
\end{gathered}
$$

Let $\delta_{1}=\min \left(\delta-\delta_{0}, \delta\right)$ and $\delta_{*}=\max \left(1, \delta_{0} / \delta_{1}\right)$,

$$
L_{*}=\left(\frac{10}{9}\right)^{4} \frac{2 E L M_{3}}{m}, \quad J_{*}=\frac{20}{9} L M_{3} L_{*}^{\delta_{*}} .
$$

Choose $\gamma_{0}$ sufficiently small so that

$$
J_{0}=\gamma_{0}^{-\frac{1}{\tau+1}} \geq J_{*},
$$

and thus $J_{v} \geq J_{0} \geq J_{*}$ for all $v \geq 0$. Now we consider a fixed $\dot{\mathfrak{R}}_{k i j}^{v}$ with $|k|>J_{*}$.

If $|k|<\frac{9 m_{v}}{10 E_{v}}\left|i^{d}-j^{d}\right|$, then $\dot{\Re}_{k i j}^{v}$ is empty.

If $|k| \geq \frac{9 m_{v}}{10 E_{v}}\left|i^{d}-j^{d}\right|$, we have

$$
|k| \geq \frac{1}{2}\left(\frac{9}{10}\right)^{3} \frac{m}{E}\left(i^{\delta}+j^{\delta}\right) .
$$

Fix $w_{1} \in\{-1,1\}^{n}$ such that $|k|=k \cdot w_{1}$ and write $\zeta=a w_{1}+w_{2}$ with $w_{1} \perp w_{2}$. As a function of $a$, for $t>s$,

$$
\begin{gathered}
\left.\langle k, \zeta\rangle\right|_{s} ^{t}=|k|(t-s), \\
\left.\left(\left|\Omega_{i}^{1}-\Omega_{j}^{1}\right|\right)\right|_{s} ^{t} \leq \frac{10}{9} L M_{3}\left(i^{\delta_{0}}+j^{\delta_{0}}\right)(t-s), \\
\left.\left(\left|\Omega_{v, i}^{2}-\Omega_{v, j}^{2}\right|\right)\right|_{s} ^{t} \leq\left(\frac{10}{9}\right)^{2} L M_{4}\left(i^{\delta}+j^{\delta}\right)(t-s) .
\end{gathered}
$$

We claim

$$
\frac{10}{9} L M_{3}\left(i^{\delta_{0}}+j^{\delta_{0}}\right) \leq \frac{|k|}{2} .
$$

In fact,

(1) If $i^{\delta_{1}}+j^{\delta_{1}} \geq L_{*}$, in view of (6.24) and

$$
2\left(i^{\delta_{0}}+j^{\delta_{0}}\right)\left(i^{\delta_{1}}+j^{\delta_{1}}\right) \leq i^{\delta}+j^{\delta},
$$

then (6.28) follows from

$$
|k| \geq\left(\frac{9}{10}\right)^{3} \frac{m}{E}\left(i^{\delta_{0}}+j^{\delta_{0}}\right)\left(i^{\delta_{1}}+j^{\delta_{1}}\right) \geq\left(\frac{9}{10}\right)^{3} \frac{m}{E}\left(i^{\delta_{0}}+j^{\delta_{0}}\right) L_{*}=\frac{20}{9} L M_{3}\left(i^{\delta_{0}}+j^{\delta_{0}}\right) ;
$$

(2) If $i^{\delta_{1}}+j^{\delta_{1}}<L_{*}$, then

$$
\frac{10}{9} L M_{3}\left(i^{\delta_{0}}+j^{\delta_{0}}\right) \leq \frac{10}{9} L M_{3}\left(i^{\delta_{1}}+j^{\delta_{1}}\right)^{\delta_{*}} \leq \frac{10}{9} L M_{3} L_{*}^{\delta_{*}}=\frac{J_{*}}{2} \leq \frac{|k|}{2} .
$$


This completes the proof of (6.28).

In view of (1.11) in assumption ( $\left.D^{*}\right),(6.24)$ and the fact $(9 / 10)^{6}>1 / 2$, we get

$$
\left(\frac{10}{9}\right)^{2} L M_{4}\left(i^{\delta}+j^{\delta}\right) \leq \frac{9}{20}|k| .
$$

Thus, from (6.25)-(6.28) and (6.32),

$$
\left.\left(\langle k, \zeta\rangle+\bar{\Omega}_{v, i}-\bar{\Omega}_{v, j}\right)\right|_{s} ^{t} \geq \frac{1}{20}|k|(t-s) .
$$

In the same process as (6.12)-(6.15) in Lemma 6.1, we finally obtain the estimate of lemma 6.2.

\section{Application to Derivative Nonlinear Schrödinger Equation}

In this section, using Theorem 1.1, we show the existence of quasi-periodic solutions for a class of derivative nonlinear Schrödinger equations subject to Dirichlet boundary conditions

$$
\left\{\begin{array}{l}
\mathbf{i} u_{t}+u_{x x}-M_{\sigma} u+\mathbf{i} f(u, \bar{u}) u_{x}=0, \quad(t, x) \in \mathbb{R} \times[0, \pi], \\
u(t, 0)=0=u(t, \pi),
\end{array}\right.
$$

where $M_{\sigma}$ is a real Fourier multiplier,

$$
M_{\sigma} \sin j x=\sigma_{j} \sin j x, \quad \sigma_{j} \in \mathbb{R}, \quad j \geq 1,
$$

and $f$ is analytic in some neighborhood of the origin in $\mathbb{C}^{2}$ with

$$
\overline{f(u, \bar{u})}=f(u, \bar{u}), \quad f(-u,-\bar{u})=-f(u, \bar{u}) .
$$

We study this equation as a Hamiltonian system on some suitable phase space $\mathscr{P}$. As the phase space one may take, for example, the usual Sobolev space $\mathscr{H}_{0}^{2}([0, \pi])$. The same as in [9], introducing the inner product

$$
\langle u, v\rangle=\operatorname{Re} \int_{0}^{\pi} u \bar{v} d x,
$$

then (7.1) can be written in Hamiltonian form

$$
\begin{gathered}
\frac{\partial u}{\partial t}=-\mathbf{i} \nabla H(u), \\
H(u)=\frac{1}{2} \int_{0}^{\pi}\left|u_{x}\right|^{2} d x+\frac{1}{2} \int_{0}^{\pi}\left(M_{\sigma} u\right) \bar{u} d x+\frac{1}{2} \int_{0}^{\pi} g(u, \bar{u}) u_{x} d x,
\end{gathered}
$$

where the gradient $\nabla$ is defined with respect to $\langle\cdot, \cdot\rangle$, and $g\left(z_{1}, z_{2}\right)=-\mathbf{i} \int_{0}^{z_{2}} f\left(z_{1}, \zeta\right) d \zeta$. To write it in infinitely many coordinates, we make the ansatz

$$
u=\mathscr{S} q=\sum_{j \geq 1} q_{j} \phi_{j}, \quad \phi_{j}=\sqrt{\frac{2}{\pi}} \sin j x, \quad j \geq 1 .
$$

The coordinates are taken from the Hilbert space $\ell_{\mathbb{C}}^{a, p}$ of all complex-valued sequences $q=\left(q_{1}, q_{2}, \cdots\right)$ with

$$
\|q\|_{a, p}^{2}=\sum_{j \geq 1}\left|q_{j}\right|^{2} j^{2 p} e^{2 a j}<\infty .
$$


We fix $a \geq 0$ and $p>\frac{3}{2}$ in the following. Then (7.4) can be rewritten as

$$
\dot{q}_{j}=-2 \mathbf{i} \frac{\partial H}{\partial \bar{q}_{j}}, j \geq 1
$$

with the Hamiltonian

$$
\begin{aligned}
H(q) & =\Lambda+G \\
& =\frac{1}{2} \sum_{j \geq 1}\left(j^{2}+\sigma_{j}\right)\left|q_{j}\right|^{2}+\frac{1}{2} \int_{0}^{\pi} g(\mathscr{S} q, \mathscr{S} \bar{q})(\mathscr{S} q)_{x} d x .
\end{aligned}
$$

The perturbation term $G$ has the following properties:

Lemma 7.1. For $a \geq 0$ and $p>\frac{3}{2}$, the function $G$ is analytic in some neighborhood of the origin in $\ell_{\mathbb{C}}^{a, p}$ with real value, and the Hamiltonian vector field $X_{G}$ is an analytic map from some neighborhood of the origin in $\ell_{\mathbb{C}}^{a, p}$ into $\ell_{\mathbb{C}}^{a, p-1}$ with

$$
\left\|X_{G}\right\|_{a, p-1}=O\left(\|q\|_{a, p}^{2}\right) .
$$

Proof. Set $h\left(z_{1}, z_{2}\right)=\int_{0}^{z_{2}} \int_{0}^{z_{1}} f(\eta, \zeta) d \eta d \zeta$. From $\overline{f(u, \bar{u})}=f(u, \bar{u})$, we know $h(u, \bar{u})$ is real. In view of the definition of $g\left(z_{1}, z_{2}\right)$ above,

$$
\overline{g(u, \bar{u})}=\overline{-\mathbf{i} \frac{\partial h(u, \bar{u})}{\partial u}}=\mathbf{i} \frac{\partial h(u, \bar{u})}{\partial \bar{u}} .
$$

Therefore,

$$
0=-\mathbf{i} \int_{0}^{\pi} \frac{d h}{d x} d x=-\mathbf{i} \int_{0}^{\pi}\left(\frac{\partial h}{\partial u} u_{x}+\frac{\partial h}{\partial \bar{u}} \bar{u}_{x}\right) d x=\int_{0}^{\pi}\left(g(u, \bar{u}) u_{x}-\overline{g(u, \bar{u})} \bar{u}_{x}\right) d x=2(G-\bar{G}) .
$$

This illustrates that $G$ is real valued. In view of $G$ in (7.9), we have

$$
\frac{\partial G}{\partial \bar{q}_{j}}=-\frac{\mathbf{i}}{2} \int_{0}^{\pi} f(u, \bar{u}) u_{x} \phi_{j} d x, \quad u=\mathscr{S} q .
$$

From $f(-u,-\bar{u})=-f(u, \bar{u})$, we know that $f(0,0)=0$ and $f(u, \bar{u}) u_{x}$ can be expanded as Fourier sine series. Thus the components of the gradient $G_{\bar{q}}$ are the Fourier sine coefficients of $f(u, \bar{u}) u_{x}$. Now the estimate (7.10) can be obtained in the same way as that of Lemma 3 in [9].

Pick a set

as $n$ basic modes. We assume

$$
J=\left\{j_{1}<j_{2}<\cdots<j_{n}\right\} \subset \mathbb{N}
$$

$$
\left\{\begin{array}{l}
\sigma_{j_{b}}=\xi_{b}, \quad b=1, \cdots, n, \\
\sigma_{j}=0, \quad j \notin J
\end{array}\right.
$$

and take $\xi:=\left(\xi_{1}, \cdots, \xi_{n}\right) \in \Pi \subset \mathbb{R}^{n}$ as parameters, where $\Pi$ is a closed bounded set of positive Lebesgue measure. We introduce symplectic polar and real coordinates $(x, y, u, v)$ by setting

$$
\left\{\begin{array}{l}
q_{j_{b}}=\sqrt{2\left(I_{b}+y_{b}\right)} e^{-\mathbf{i} x_{b}}, \quad b=1, \cdots, n, \\
q_{j}=u_{j}-\mathbf{i} v_{j}, \quad j \notin J,
\end{array}\right.
$$

where $I=\left(I_{1}, \cdots, I_{n}\right)$ is fixed. Then we have

$$
-\frac{\mathbf{i}}{2} \sum_{j \geq 1} d q_{j} \wedge d \bar{q}_{j}=\sum_{1 \leq b \leq n} d y_{b} \wedge d x_{b}+\sum_{j \notin J} d u_{j} \wedge d v_{j} .
$$


Therefore, up to a constant term, the Hamiltonian (7.9) can be rewritten as

$$
\begin{aligned}
H & =N+P \\
& =\sum_{1 \leq b \leq n} \omega_{b} y_{b}+\frac{1}{2} \sum_{j \notin J} \Omega_{j}\left(u_{j}^{2}+v_{j}^{2}\right)+G(q(x, y, u, v))
\end{aligned}
$$

with symplectic structure $\sum_{1 \leq b \leq n} d y_{b} \wedge d x_{b}+\sum_{j \notin J} d u_{j} \wedge d v_{j}$, where

$$
\begin{gathered}
\omega_{b}=j_{b}^{2}+\xi_{b}, \quad b=1, \cdots, n, \\
\Omega_{j}=j^{2}, \quad j \notin J .
\end{gathered}
$$

It's obvious that the tangential frequencies $\omega=\left(\omega_{1}, \cdots, \omega_{n}\right)$ and normal frequencies $\Omega=\left(\Omega_{j}: j \notin J\right)$ satisfy the assumptions (A) (B) (C) (D) in Theorem 1.1. From Lemma 7.1 above, we know there exists $r>0$, such that, for every fixed $I$ satisfying $|I|=O\left(r^{2}\right)$, the Hamiltonian vector field $X_{P}$ is real analytic from $D(1, r)$ to $\mathscr{P}^{a, p-1}$ with

$$
\left\|X_{P}\right\|_{r, a, p-1, D(1, r)}=O(r) .
$$

Moreover, since $X_{P}$ is independent of $\xi$, we know

$$
\left\|X_{P}\right\|_{r, a, p-1, D(1, r) \times \Pi}^{\operatorname{lip}}=0 .
$$

Therefore, defining $\varepsilon$ as (1.7), we have

$$
\varepsilon=O(r) .
$$

We simply assume $|\Pi|=O(1)$ and fix $\beta=1 / 5$. Then by letting $\alpha=O\left(r^{2 / 3}\right)$, the inequality in (1.7) is satisfied when $r$ is small enough. Now Theorem 1.1 yields the following

Theorem 7.2. Consider a family of derivative nonlinear Schrödinger equation (7.1) parameterized by the Fourier multiplier $M_{\sigma}$ with $\sigma=\sigma(\xi)$ defined by (7.14). Then for any $0<\varepsilon \ll 1$, there is a subset $\Pi_{\varepsilon} \subset \Pi$ with

$$
\left|\Pi \backslash \Pi_{\varepsilon}\right|=O\left(\varepsilon^{2 / 3}\right),
$$

such that for every $\xi \in \Pi_{\varepsilon}$, the equation has a smooth quasi-periodic solution of the form

$$
u(t, x)=\sum_{j \geq 1} \tilde{q}_{j}(t) \phi_{j}(x)
$$

where $\left\{\tilde{q}_{j}\right\}_{j \geq 1}$ are quasi-periodic functions with frequencies $\omega^{\prime}:=\left(\omega_{1}^{\prime}, \cdots, \omega_{n}^{\prime}\right)$. Moreover,

$$
\left|\omega^{\prime}-\omega\right|=O(\varepsilon)
$$

and for every non-negative integer $v$, there exists a positive constant c depending on $v$ such that

$$
\begin{gathered}
\left|\frac{d^{v} \tilde{q}_{j}}{d t^{v}}\right| \leq c \varepsilon, \quad j \in J, \\
\sum_{j \notin J} e^{2 a j} j^{2 p}\left|\frac{d^{v} \tilde{q}_{j}}{d t^{v}}\right|^{2} \leq c \varepsilon^{7 / 3} .
\end{gathered}
$$


Remarks. 1. The estimate (7.25) follows from (1.10) in Theorem 1.1. From (1.9) in Theorem 1.1, we know

$$
\begin{aligned}
& \left(\max _{1 \leq b \leq n}\left|\frac{d^{v}\left(\tilde{x}_{b}-\theta_{b}-\omega_{b}^{\prime} t\right)}{d t^{v}}\right|\right)+\left(\max _{1 \leq b \leq n}\left|\frac{d^{v} \tilde{y}_{b}}{d t^{v}}\right|\right) r^{-2}+\left(\sum_{j \notin J} e^{2 a j} j^{2 p}\left|\frac{d^{v} \tilde{q}_{j}}{d t^{\nu}}\right|^{2}\right)^{1 / 2} r^{-1} \\
= & O\left(\varepsilon^{\frac{1}{1+\beta}} / \alpha\right)=O\left(\varepsilon^{1 / 6}\right),
\end{aligned}
$$

where $\tilde{q}_{j_{b}}=\sqrt{2\left(I_{b}+\tilde{y}_{b}\right)} e^{\mathbf{i} \tilde{x}_{b}}, 1 \leq b \leq n$ and $\theta=\left(\theta_{1}, \ldots, \theta_{n}\right) \in \mathbb{T}^{n}$. Furthermore,

$$
\begin{gathered}
\frac{d^{v}\left(\tilde{x}_{b}-\theta_{b}-\omega_{b}^{\prime} t\right)}{d t^{v}}=O\left(\varepsilon^{1 / 6}\right), \quad \frac{d^{v} \tilde{y}_{b}}{d t^{v}}=O\left(\varepsilon^{13 / 6}\right), \\
\left(\sum_{j \notin J} e^{2 a j} j^{2 p}\left|\frac{d^{v} \tilde{q}_{j}}{d t^{v}}\right|^{2}\right)^{1 / 2}=O\left(\varepsilon^{7 / 6}\right) .
\end{gathered}
$$

The estimate (7.26) follows from (7.28) and $I=O\left(\varepsilon^{2}\right)$. The estimate (7.27) follows from (7.29).

2. Under Dirichlet boundary conditions, in order to get the Hamiltonian (7.9) of discrete form, one needs to develop $u=u(t, x)$ into Fourier sine series (7.6). Once it is done, the nonlinearity must be developed into Fourier sine series in proving the regularity of nonlinear Hamiltonian vector field (see Lemma 7.1). This excludes the usual nonlinearity $\left(|u|^{2} u\right)_{x}$. Naturally, one expects to investigate the DNLS equation with nonlinearity $\left(|u|^{2} u\right)_{x}$ subject to periodic boundary conditions. In this case, one must extend Lemma 3.1 to higher dimension. In present time, this is an open problem.

\section{Application to Perturbed Benjamin-Ono Equation}

The Benjamin-Ono equation describes the evolution of the interface between two inviscid fluids under some physical conditions (see [3]). Under periodic boundary conditions it reads

$$
u_{t}+\mathscr{H} u_{x x}-u u_{x}=0, \quad(t, x) \in \mathbb{R} \times \mathbb{T},
$$

where $u$ is real-valued and $\mathscr{H}$ is the Hilbert transform defined for $2 \pi$-periodic functions with mean value zero by

$$
\widehat{\mathscr{H}(f)}(0)=0, \quad \widehat{\mathscr{H}(f)}(j)=-\mathbf{i s g n}(j) \hat{f}(j), \quad j \in \mathbb{Z} \backslash\{0\} .
$$

The Benjamin-Ono equation is an integrable system (see [1]). For the global well-posedness of the Cauchy problem of the above equation, see [13], [14]. In the first subsection, we transform the Benjamin-Ono Hamiltonian into its Birkhoff normal form up to order four. In the second subsection, by using Theorem 1.2, we prove there are many KAM tori and thus quasi-periodic solutions for the above equation with small Hamiltonian perturbations.

\subsection{Birkhoff Normal Form}

We introduce for any $N>3 / 2$ the phase space

$$
\mathscr{H}_{0}^{N}=\left\{u \in L^{2}(\mathbb{T} ; \mathbb{R}): \hat{u}(0)=0, \quad\|u\|_{N}^{2}=\sum_{j \in \mathbb{Z} \backslash\{0\}}|j|^{2 N}|\hat{u}(j)|^{2}<\infty\right\}
$$

of real valued functions on $\mathbb{T}$, where

$$
\hat{u}(j)=\int_{0}^{2 \pi} u(x) e_{-j}(x) d x, \quad e_{j}(x)=\frac{1}{\sqrt{2 \pi}} e^{\mathbf{i} j x}
$$


Under the standard inner product on $L^{2}(\mathbb{T} ; \mathbb{R})$, equation $(8.1)$ can be written in Hamiltonian form

$$
\frac{\partial u}{\partial t}=-\frac{d}{d x} \frac{\partial H}{\partial u}
$$

with Hamiltonian

$$
H(u)=\frac{1}{2} \int_{\mathbb{T}} u\left(\mathscr{H} u_{x}\right) d x-\frac{1}{6} \int_{\mathbb{T}} u^{3} d x .
$$

To write it in infinitely many coordinates, we make the ansatz

$$
u(t, x)=\sum_{j \neq 0} \gamma_{j} q_{j}(t) e_{j}(x)
$$

where $\gamma_{j}=\sqrt{|j|}$. The coordinates are taken from the Hilbert space $\ell_{N+1 / 2}$ of all complex-valued sequences $q=\left(q_{j}\right)_{j \neq 0}$ with

$$
\|q\|_{N+1 / 2}^{2}=\sum_{j \neq 0}\left|q_{j}\right|^{2} j^{2 N+1}<\infty, \quad q_{-j}=\bar{q}_{j} .
$$

Then (8.3) can be rewritten as

$$
\dot{q}_{j}=-\mathbf{i} \sigma_{j} \frac{\partial H}{\partial q_{-j}}, \quad \sigma_{j}=\left\{\begin{array}{l}
1, j \geq 1 \\
-1, j \leq-1
\end{array}\right.
$$

with the Hamiltonian

$$
H(q)=\Lambda+G=\sum_{j \geq 1} j^{2}\left|q_{j}\right|^{2}-\frac{1}{6 \sqrt{2 \pi}} \sum_{j+k+l=0} \gamma_{j} \gamma_{k} \gamma_{l} q_{j} q_{k} q_{l}
$$

The function $G$ is analytic in $\ell_{N+1 / 2}$ with real value, and the Hamiltonian vector field $X_{G}$ is an analytic map from $\ell_{N+1 / 2}$ into $\ell_{N-1 / 2}$ with

$$
\left\|X_{G}\right\|_{N-1 / 2}=O\left(\|q\|_{N+1 / 2}^{2}\right)
$$

In the following theorem, we transform the above Hamiltonian into its Birkhoff normal form up to order four.

Theorem 8.1. There exists a real analytic symplectic coordinate transformation $\Phi$ defined in a neighborhood of the origin of $\ell_{N+1 / 2}$ which transforms the above Hamiltonian $H$ into its Birkhoff normal form up to order four. More precisely,

$$
H \circ \Phi=\Lambda+B+R
$$

with

$$
\begin{gathered}
B=-\frac{1}{8 \pi} \sum_{j, k \geq 1} \min (j, k)\left|q_{j}\right|^{2}\left|q_{k}\right|^{2}, \\
\left\|X_{R}\right\|_{N-1 / 2}=O\left(\|q\|_{N+1 / 2}^{4}\right) .
\end{gathered}
$$

Proof. (1). The first step is to eliminate the three order term $G$. Define $F^{3}=\sum_{j, k, l \neq 0} F_{j k l}^{3} q_{j} q_{k} q_{l}$ by

$$
i F_{j k l}^{3}= \begin{cases}\frac{1}{6 \sqrt{2 \pi}} \frac{\gamma_{j} \gamma_{\gamma_{1}}}{\lambda_{j}+\lambda_{k}+\lambda_{l}}, & \text { for } j+k+l=0 \\ 0, & \text { otherwise }\end{cases}
$$


where $\lambda_{j}=\sigma_{j} j^{2}$. Then we have

$$
\left\{\Lambda, F^{3}\right\}+G=0,
$$

where $\{\cdot, \cdot\}$ is Poisson bracket with respect to the symplectic structure $-\mathbf{i} \sum_{j \geq 1} d q_{j} \wedge d q_{-j}$. Letting $\Phi_{1}=X_{F^{3}}^{1}$, then

$$
\begin{aligned}
H \circ \Phi_{1} & =\left.H \circ X_{F^{3}}^{t}\right|_{t=1} \\
& =\Lambda+\left\{\Lambda, F^{3}\right\}+\int_{0}^{1}(1-t)\left\{\left\{\Lambda, F^{3}\right\}, F^{3}\right\} \circ X_{F^{3}}^{t} d t+G+\int_{0}^{1}\left\{G, F^{3}\right\} \circ X_{F^{3}}^{t} d t \\
& =\Lambda+\int_{0}^{1} t\left\{G, F^{3}\right\} \circ X_{F^{3}}^{t} d t \\
& =\Lambda+\frac{1}{2}\left\{G, F^{3}\right\}+\frac{1}{2} \int_{0}^{1}\left(1-t^{2}\right)\left\{\left\{G, F^{3}\right\}, F^{3}\right\} \circ X_{F^{3}}^{t} d t .
\end{aligned}
$$

The j-th element of vector field $X_{F^{3}}$ reads explicitly

$$
-\mathbf{i} \sigma_{j} \frac{\partial F^{3}}{\partial q_{-j}}=-\mathbf{i} \sigma_{j} \sum_{k+l=j} 3 F_{(-j) k l}^{3} q_{k} q_{l}=\frac{-\sigma_{j}}{2 \sqrt{2 \pi}} \sum_{k+l=j} \frac{\gamma_{j} \gamma_{k} \gamma_{l}}{\lambda_{-j}+\lambda_{k}+\lambda_{l}} q_{k} q_{l} .
$$

For any $j, k, l \neq 0$ with $j+k+l=0$,

$$
\lambda_{j}+\lambda_{k}+\lambda_{l}=2 j k l / \max \{|j|,|k|,|l|\} .
$$

Thus,

$$
\left|\frac{\gamma_{k+l} \gamma_{k} \gamma_{l}}{\lambda_{-k-l}+\lambda_{k}+\lambda_{l}}\right|=\frac{\max \{|k+l|,|k|,|l|\}}{2 \sqrt{|(k+l) k l|}} \leq \frac{\sqrt{2}}{2} .
$$

Hence we have the estimate

$$
\left|-\mathbf{i} \sigma_{j} \frac{\partial F^{3}}{\partial q_{-j}}\right| \leq \frac{1}{4 \sqrt{\pi}} \sum_{k+l=j}\left|q_{k}\right|\left|q_{l}\right|=\frac{1}{4 \sqrt{\pi}} g_{-j},
$$

where $g_{-j}$ stands for the sum $\sum_{k+l=j}\left|q_{k}\right|\left|q_{l}\right|$. Obviously, $g=\left(g_{j}\right)_{j \neq 0}$ is the two-fold convolution of $w=\left(\left|q_{j}\right|\right)_{j \neq 0}$, that is, $g=w * w$. Thus, for any $r>1 / 2$,

$$
\left\|X_{F^{3}}\right\|_{r} \leq \frac{1}{4 \sqrt{\pi}}\|g\|_{r} \leq c\|w\|_{r}^{2}=c\|q\|_{r}^{2},
$$

where $c>0$ depends only on $r$. This establishes the regularity of the vector field $X_{F^{3}}$.

(2). The second step is to normalize the four order term $\frac{1}{2}\left\{G, F^{3}\right\}$ in (8.15). By a simple calculation, we have

$$
\begin{aligned}
\frac{1}{2}\left\{G, F^{3}\right\}= & -\frac{\mathbf{i}}{2} \sum_{j \neq 0} \sigma_{j} \frac{\partial G}{\partial q_{j}} \frac{\partial F^{3}}{\partial q_{-j}} \\
= & \frac{1}{16 \pi} \sum_{j \neq 0} \sigma_{j}\left(\sum_{k+l=-j} \gamma_{j} \gamma_{k} \gamma_{l} q_{k} q_{l}\right)\left(\sum_{m+n=j} \frac{\gamma_{-j} \gamma_{m} \gamma_{n}}{\lambda_{-j}+\lambda_{m}+\lambda_{n}} q_{m} q_{n}\right) \\
= & \frac{1}{16 \pi} \sum_{\substack{k, l, m, n \neq 0 \\
k+l+m+n=0 \\
k+l \neq 0}} \frac{(m+n) \gamma_{k} \gamma_{l} \gamma_{m} \gamma_{n}}{-\lambda_{m+n}+\lambda_{m}+\lambda_{n}} q_{k} q_{l} q_{m} q_{n} . \\
& \sum^{l \neq m+n},
\end{aligned}
$$


Let $B$ consist of all terms with $k+m=0$ or $k+n=0$ in (8.21). Then write $B$ explicitly,

$$
\begin{aligned}
B & =\frac{1}{16 \pi} \sum_{k \neq 0} \frac{-2 k^{3}}{\lambda_{2 k}-2 \lambda_{k}}\left|q_{k}\right|^{4}+\frac{1}{8 \pi} \sum_{k, l, k \pm l \neq 0} \frac{-(k+l)|k l|}{\lambda_{k+l}-\lambda_{k}-\lambda_{l}}\left|q_{k}\right|^{2}\left|q_{l}\right|^{2} \\
& =-\frac{1}{16 \pi} \sum_{k \neq 0}|k|\left|q_{k}\right|^{4}-\frac{1}{16 \pi} \sum_{k, l, k \pm l \neq 0} \sigma_{k l} \max \{|k|,|l|,|k+l|\}\left|q_{k}\right|^{2}\left|q_{l}\right|^{2} \\
& =-\frac{1}{8 \pi} \sum_{k \geq 1} k\left|q_{k}\right|^{4}-\frac{1}{4 \pi} \sum_{k>l \geq 1} l\left|q_{k}\right|^{2}\left|q_{l}\right|^{2}
\end{aligned}
$$

which is (8.11). Let $Q=\frac{1}{2}\left\{G, F^{3}\right\}-B$. We will find a coordinate transformation $\Phi_{2}=X_{F^{4}}^{1}$ to eliminate $Q$. In complete analogy to the first step we let

$$
\begin{gathered}
F^{4}=\sum_{k, l, m, n \neq 0} F_{k l m n}^{4} q_{k} q_{l} q_{m} q_{n}, \\
i F_{k l m n}^{4}=\left\{\begin{array}{lc}
-\frac{1}{16 \pi} \frac{(m+n) \gamma_{k} \gamma_{l} \gamma_{m} \gamma_{n}}{\left(-\lambda_{m+n}+\lambda_{m}+\lambda_{n}\right)\left(\lambda_{k}+\lambda_{l}+\lambda_{m}+\lambda_{n}\right)}, & \text { for } \begin{array}{l}
k+l+m+n=0 \\
k+l, k+m, k+n \neq 0
\end{array} \\
0, & \text { otherwise. }
\end{array},\right.
\end{gathered}
$$

Then we have

$$
\left\{\Lambda, F^{4}\right\}+Q=0 .
$$

In order to complete the second step, we only need to establish the regularity of the vector field $X_{F^{4}}$. We claim that for $f_{k l m n}:=\frac{-\lambda_{m+n}+\lambda_{m}+\lambda_{n}}{m+n}\left(\lambda_{k}+\lambda_{l}+\lambda_{m}+\lambda_{n}\right)$ with $k+l+m+n=0$ and $k, l, m, n, k+$ $l, k+m, k+n \neq 0$,

$$
\left|f_{k l m n}\right| \geq \frac{1}{2} \max \{|k|,|l|,|m|,|n|\} .
$$

We prove this claim in four cases. Ahead of the proof, we give a simple inequality for two positive integers $a, b$ :

$$
2 a b \geq a+b,
$$

which will be frequently used. Without loss of generality, we assume $|k| \geq|l|$ and $|m| \geq|n|$ because of their symmetry in $f_{k l m n}$.

(1) $l, m, n$ have the opposite sign of $k$. Without loss of generality, we assume $k<0$ and $l, m, n>0$. In this case, we have

$$
\begin{gathered}
\left|-\lambda_{m+n}+\lambda_{m}+\lambda_{n}\right|=2|m n| \geq|m+n| \\
\left|\lambda_{k}+\lambda_{l}+\lambda_{m}+\lambda_{n}\right|=2(\operatorname{lm}+\ln +m n) \geq 2(\operatorname{lm}+\ln )=2 l(|k|-l) \geq|k|,
\end{gathered}
$$

which leads to (8.26).

(2) $k, l, n$ have the opposite sign of $m$. Without loss of generality, we assume $m<0$ and $k, l, n>0$. In this case, we have

$$
\begin{gathered}
\left|-\lambda_{m+n}+\lambda_{m}+\lambda_{n}\right|=2|(m+n) n| \geq|m+n|, \\
\left|\lambda_{k}+\lambda_{l}+\lambda_{m}+\lambda_{n}\right|=2(k l+k n+\ln ) \geq 2(k n+l n)=2(|m|-n) n \geq|m|,
\end{gathered}
$$

which leads to (8.26). 
(3) $k, l$ have the same sign while $m, n$ have the same sign. Without loss of generality, we assume $k, l>0$ and $m, n<0$. Here (8.26) follows from the following inequalities:

$$
\begin{gathered}
\left|\frac{-\lambda_{m+n}+\lambda_{m}+\lambda_{n}}{m+n}\right|=\left|\frac{2 m n}{m+n}\right| \geq|n|, \\
|n|\left|\lambda_{k}+\lambda_{l}+\lambda_{m}+\lambda_{n}\right|=2|n|(k-|n|)|k-| m|| \geq k|k-| m|| \geq \frac{1}{2} \max \{k,|m|\} .
\end{gathered}
$$

(4) $k, n$ have the same sign while $l, m$ have the same sign. Without loss of generality, we assume $k, n>0$ and $l, m<0$. Here (8.26) follows from the following inequalities:

$$
\begin{gathered}
\left|\frac{-\lambda_{m+n}+\lambda_{m}+\lambda_{n}}{m+n}\right|=2 n, \\
2 n\left|\lambda_{k}+\lambda_{l}+\lambda_{m}+\lambda_{n}\right|=4 n(|m|-n)|| m|-k| \geq 2|m||k-| m|| \geq \max \{k,|m|\} .
\end{gathered}
$$

Finally, the proof of (8.26) is finished. From (8.26) we get

$$
\left|F_{k l m n}^{4}\right| \leq \frac{\gamma_{k} \gamma_{l} \gamma_{m} \gamma_{n}}{8 \pi \max \{|k|,|l|,|m|,|n|\}}
$$

The $\mathrm{j}$-th element of vector field $X_{F^{4}}$ explicitly reads

$$
-\mathbf{i} \sigma_{j} \frac{\partial F^{4}}{\partial q_{-j}}=-\mathbf{i} \sigma_{j} \sum_{l+m+n=j}\left(F_{(-j) l m n}^{4}+F_{l(-j) m n}^{4}+F_{l m(-j) n}^{4}+F_{l m n(-j)}^{4}\right) q_{l} q_{m} q_{n} .
$$

Hence we have the estimate

$$
\left|-\mathbf{i} \sigma_{j} \frac{\partial F^{4}}{\partial q_{-j}}\right| \leq \frac{1}{2 \pi \gamma_{j}} \sum_{l+m+n=j} \gamma_{l} \gamma_{m} \gamma_{n}\left|q_{l} q_{m} q_{n}\right| \leq \frac{1}{2 \pi \gamma_{j}} g_{-j}
$$

where $g_{-j}$ stands for the sum $\sum_{l+m+n=j} \gamma_{l} \gamma_{m} \gamma_{n}\left|q_{l}\right|\left|q_{m}\right|\left|q_{n}\right|$. Obviously, $g=\left(g_{j}\right)_{j \neq 0}$ is the three-fold convolution of $w=\left(\gamma_{j}\left|q_{j}\right|\right)_{j \neq 0}$, that is $g=w * w * w$. Thus, for any $r>1$,

$$
\left\|X_{F^{4}}\right\|_{r} \leq \frac{1}{2 \pi}\|g\|_{r-1 / 2} \leq c\|w\|_{r-1 / 2}^{3}=c\|q\|_{r}^{3},
$$

where $c>0$ depends only on $r$. This establishes the regularity of the vector field $X_{F^{4}}$, and finishes the proof of Theorem 8.1.

\subsection{Quasi-periodic Solutions for Perturbed Benjamin-Ono Equation}

Here is our main result in this section:

Theorem 8.2. Consider the Benjamin-Ono equation (8.1) with a small Hamiltonian perturbation, written in the Hamiltonian form

$$
\frac{\partial u}{\partial t}=-\frac{d}{d x}\left(\frac{\partial H}{\partial u}+\varepsilon \frac{\partial K}{\partial u}\right)
$$

where the Hamiltonian $H$ is defined by (8.4), and the Hamiltonian $K$ is real analytic in a complex neighborhood $V$ of the origin in $\mathscr{H}_{0, \mathbb{C}}^{N}$, which is the complexification of $\mathscr{H}_{0}^{N}, N>3 / 2$. Moreover, $K$ satisfies the regularity condition

$$
\frac{\partial K}{\partial u}: \quad V \rightarrow \mathscr{H}_{0, \mathbb{C}}^{N}, \quad\left\|\frac{\partial K}{\partial u}\right\|_{N, V} \leq 1 .
$$

Then, for each index set $J=\left\{j_{1}<j_{2}<\cdots<j_{n}\right\} \subset \mathbb{N}$, there exists an $\varepsilon_{0}>0$ depending only on $J, N$ and the size of $V$, such that for $\varepsilon<\varepsilon_{0}$, the equation has uncountable quasi-periodic solutions with frequency vector close to $\left(j_{1}^{2}, \cdots, j_{n}^{2}\right)$. 
Proof. Set the perturbed Hamiltonian $\tilde{H}=H+\varepsilon K$. Then, by the transformation $\Phi$ in Theorem 8.1, we get the new Hamiltonian, still denoted by $\tilde{H}$,

$$
\tilde{H}=\Lambda+B+R+\varepsilon K \circ \Phi,
$$

which is analytic in some neighborhood $U$ of the origin of $\ell_{N+1 / 2}$ with $\Lambda$ in (8.8), $B$ in (8.11), $R$ satisfying (8.12) and the last term satisfying

$$
\left\|X_{K \circ \Phi}\right\|_{N-1 / 2, U} \leq 2 .
$$

We introduce symplectic polar and real coordinates $(x, y, u, v)$ by setting

$$
\left\{\begin{array}{l}
q_{j_{b}}=\sqrt{\xi_{b}+y_{b}} e^{-\mathbf{i} x_{b}}, \quad q_{-j_{b}}=\sqrt{\xi_{b}+y_{b}} e^{\mathbf{i} x_{b}}, \quad b=1, \cdots, n, \\
q_{j}=\frac{1}{\sqrt{2}}\left(u_{j}-\mathbf{i} v_{j}\right), \quad q_{-j}=\frac{1}{\sqrt{2}}\left(u_{j}+\mathbf{i} v_{j}\right), \quad j \in \mathbb{N}_{*}:=\mathbb{N} \backslash J,
\end{array}\right.
$$

where $\xi=\left(\xi_{1}, \cdots, \xi_{n}\right) \in \mathbb{R}_{+}^{n}$. Then

$$
\begin{aligned}
\Lambda= & \sum_{1 \leq b \leq n} j_{b}^{2}\left(\xi_{b}+y_{b}\right)+\frac{1}{2} \sum_{j \in \mathbb{N}_{*}} j^{2}\left(u_{j}^{2}+v_{j}^{2}\right), \\
-8 \pi B= & \sum_{1 \leq b, b^{\prime} \leq n} \min \left(j_{b}, j_{b^{\prime}}\right)\left(\xi_{b}+y_{b}\right)\left(\xi_{b^{\prime}}+y_{b^{\prime}}\right) \\
& +\sum_{1 \leq b \leq n, j \in \mathbb{N}_{*}} \min \left(j_{b}, j\right)\left(\xi_{b}+y_{b}\right)\left(u_{j}^{2}+v_{j}^{2}\right) \\
& +\frac{1}{4} \sum_{j, j^{\prime} \in \mathbb{N}_{*}} \min \left(j, j^{\prime}\right)\left(u_{j}^{2}+v_{j}^{2}\right)\left(u_{j^{\prime}}^{2}+v_{j^{\prime}}^{2}\right) .
\end{aligned}
$$

Thus the new Hamiltonian, still denoted by $\tilde{H}$, up to a constant depending only on $\xi$, is given by

$$
\tilde{H}=N+P=\sum_{1 \leq b \leq n} \omega_{b} y_{b}+\frac{1}{2} \sum_{j \in \mathbb{N}_{*}} \Omega_{j}\left(u_{j}^{2}+v_{j}^{2}\right)+Q+R+\varepsilon K,
$$

with symplectic structure $\sum_{1 \leq b \leq n} d y_{b} \wedge d x_{b}+\sum_{j \in \mathbb{N}_{*}} d u_{j} \wedge d v_{j}$, where

$$
\begin{gathered}
\omega_{b}=j_{b}^{2}-\frac{1}{4 \pi} \sum_{1 \leq b^{\prime} \leq n} \min \left(j_{b}, j_{b^{\prime}}\right) \xi_{b^{\prime}}, \\
\Omega_{j}=j^{2}-\frac{1}{4 \pi} \sum_{1 \leq b \leq n} \min \left(j_{b}, j\right) \xi_{b}, \\
Q=-\frac{1}{8 \pi} \sum_{1 \leq b, b^{\prime} \leq n} \min \left(j_{b}, j_{b^{\prime}}\right) y_{b} y_{b^{\prime}}-\frac{1}{8 \pi} \sum_{1 \leq b \leq n, j \in \mathbb{N}_{*}} \min \left(j_{b}, j\right) y_{b}\left(u_{j}^{2}+v_{j}^{2}\right) \\
-\frac{1}{32 \pi} \sum_{j, j^{\prime} \in \mathbb{N}_{*}} \min \left(j, j^{\prime}\right)\left(u_{j}^{2}+v_{j}^{2}\right)\left(u_{j^{\prime}}^{2}+v_{j^{\prime}}^{2}\right) .
\end{gathered}
$$

Set

$$
\Pi=\left\{\xi \in \mathbb{R}_{+}^{n}:|\xi| \leq \varepsilon^{2 / 5}\right\} .
$$

In the following we check the assumptions (A) (B) (C) and (D*). 
In view of (8.39), we know that $\xi \mapsto \omega$ is an affine transformation from $\Pi$ to $\mathbb{R}^{n}$. Regarding $\omega$ as $n$-dimensional column vector,

$$
\omega=\breve{\omega}-\frac{1}{4 \pi} A \xi=\left(\begin{array}{c}
j_{1}^{2} \\
j_{2}^{2} \\
j_{3}^{2} \\
\vdots \\
j_{n}^{2}
\end{array}\right)-\frac{1}{4 \pi}\left(\begin{array}{ccccc}
j_{1} & j_{1} & j_{1} & \cdots & j_{1} \\
j_{1} & j_{2} & j_{2} & \cdots & j_{2} \\
j_{1} & j_{2} & j_{3} & \cdots & j_{3} \\
\vdots & \vdots & \vdots & \ddots & \vdots \\
j_{1} & j_{2} & j_{3} & \cdots & j_{n}
\end{array}\right)\left(\begin{array}{c}
\xi_{1} \\
\xi_{2} \\
\xi_{3} \\
\vdots \\
\xi_{n}
\end{array}\right)
$$

where $\check{\omega}$ is a $n$-dimensional column vector with its $b$-th element $\check{\omega}_{b}=j_{b}^{2}$ and $A$ is a $n \times n$ matrix with its $(b, m)$-th element $A_{b m}=\min \left(j_{b}, j_{m}\right)$. Let

$$
T=\left(\begin{array}{ccccc}
1 & -1 & & & \\
& 1 & -1 & & \\
& 1 & \ddots & \\
& & & \ddots & -1 \\
& & & & 1
\end{array}\right)\left(\begin{array}{ccccc}
1 & & & & \\
-\frac{j_{1}}{j_{2}-j_{1}} & 1 & & & \\
& -\frac{j_{2}-j_{1}}{j_{3}-j_{2}} & 1 & & \\
& & \ddots & \ddots & \\
& & & -\frac{j_{n-1}-j_{n-2}}{j_{n}-j_{n-1}} & 1
\end{array}\right) .
$$

Then by a simple calculation, we have $A T=\tilde{A}:=\operatorname{diag}\left(j_{b}-j_{b-1}: 1 \leq b \leq n\right)$ (here $\left.j_{0}=0\right)$. Thus,

$$
\operatorname{det}\left(\frac{\partial \omega}{\partial \xi}\right)=\left(-\frac{1}{4 \pi}\right)^{n} j_{1}\left(j_{2}-j_{1}\right)\left(j_{3}-j_{2}\right) \cdots\left(j_{n}-j_{n-1}\right) \neq 0
$$

This implies that the assumption (A) is fulfilled with positive constant $M_{1}$ and $L$ only depending on the set $J$.

In view of (8.40), we know

$$
\Omega_{j}=j^{2}-\frac{1}{4 \pi} B_{j} \xi
$$

where $B_{j}$ is a $n$-dimensional row vector with its $m$-th element $B_{j m}=\min \left(j, j_{m}\right)$. It's obvious that $\Omega_{j} \approx j^{2}$, thus, the assumption (B) is fulfilled with $d=2$ and $m=1 / 2$.

From (8.46), we get

$$
\left|\Omega_{j}\right|_{\Pi}^{\text {lip }}=\frac{1}{4 \pi} \sum_{1 \leq m \leq n} B_{j m}=\frac{1}{4 \pi} \sum_{1 \leq m \leq n} \min \left(j, j_{m}\right) .
$$

Thus,

$$
|\Omega|_{-1, \Pi}^{\operatorname{lip}}=\sup _{j \in \mathbb{N}_{*}} j^{-1}\left|\Omega_{j}\right|_{\Pi}^{\operatorname{lip}} \leq \frac{n}{4 \pi},
$$

that is, assumption (C) is fulfilled with $\delta=1$ and $M_{2}=\frac{n}{4 \pi}$.

Moreover, taking $\delta_{0}=0$ and letting $\Omega_{j}^{1}=\Omega_{j}, \Omega_{j}^{2}=0$ in the assumption (D*), then from (8.47) we have

$$
\left|\Omega^{1}\right|_{-\delta_{0}, \Pi}^{\text {lip }}=\sup _{j \in \mathbb{N}_{*}}\left|\Omega_{j}\right|_{\Pi}^{\text {lip }}=\frac{1}{4 \pi} \sum_{1 \leq m \leq n} j_{m}:=M_{3},
$$

which only depends on $J$. On the other hand, since $\Omega^{2}=0$, the positive constant $M_{4}$ can be chosen arbitrarily small. Therefore, we can choose it small enough such that (1.11) is fulfilled. Thus the second part of the assumption $\left(\mathrm{D}^{*}\right)$ holds true.

In view of (8.46), regarding $\Omega$ as an infinite dimensional column vector with its index $j \in \mathbb{N}_{*}$, we have

$$
\Omega=\check{\Omega}-\frac{1}{4 \pi} B \xi
$$


where $\check{\Omega}$ is an infinite dimensional column vector with its $j$-th element $\check{\Omega}_{j}=j^{2}$ and $B$ is a $\infty \times n$ matrix with its $j$-th row $B_{j}$. For the first part of the assumption ( $\left.\mathrm{D}^{*}\right)$, regarding $k$ and $l$ as $n$ dimensional and infinite dimensional row vectors respectively, we have to check for every $k \in \mathbb{Z}^{n}$ and $l \in \mathbb{Z}^{\infty}$ with $1 \leq|l| \leq 2$,

$$
k \check{\omega}+l \check{\Omega} \neq 0 \quad \text { or } \quad k A+l B \neq 0 .
$$

By a simple calculation, we have $B A^{-1}=B T \tilde{A}^{-1}=\tilde{B}$, which is a $\infty \times n$ matrix with its $j$-th row $\tilde{B}_{j}$ :

(1) $\tilde{B}_{j}=\left(\frac{j}{j_{1}}, 0, \cdots, 0\right)$ for $j<j_{1}$, only the first element nonzero;

(2) $\tilde{B}_{j}=\left(0, \cdots, 0, \frac{j_{m+1}-j}{j_{m+1}-j_{m}}, \frac{j-j_{m}}{j_{m+1}-j_{m}}, 0, \cdots, 0\right)$ for $j_{m}<j<j_{m+1}$ with $1 \leq m<n$, only the $m$-th and $(m+1)$-th elements nonzero;

(3) $\tilde{B}_{j}=(0, \cdots, 0,1)$ for $j>j_{n}$, only the last element nonzero.

Then the second inequality of $(8.51)$ is equivalent to

$$
k+l \tilde{B} \neq 0 .
$$

Set $\mathbb{N}_{*}=\mathbb{N}_{*}^{1} \cup \mathbb{N}_{*}^{2} \cup \mathbb{N}_{*}^{3}$, where $\mathbb{N}_{*}^{1}=\left\{1 \leq j<j_{1}\right\}, \mathbb{N}_{*}^{2}=\left\{j_{1} \leq j \leq j_{n}\right\} \backslash J$ and $\mathbb{N}_{*}^{3}=\left\{j>j_{n}\right\}$. Notice that for $j \in \mathbb{N}_{*}^{1} \cup \mathbb{N}_{*}^{2}$ the nonzero elements of $\tilde{B}_{j}$ are positive and less than 1 . Thus the equality (8.52) holds true for $k \in \mathbb{Z}^{n}$ and $1 \leq|l| \leq 2$ except the following three cases:

(1)

$$
l_{j}=\left\{\begin{array}{ll} 
\pm 2, & j=j_{1} / 2 \\
0, & \text { otherwise }
\end{array}, \quad k_{m}=\left\{\begin{array}{ll}
\mp 1, & m=1 \\
0, & \text { otherwise }
\end{array} ;\right.\right.
$$

(2) For a fixed $m^{\prime}$ with $1 \leq m^{\prime}<n$,

$$
l_{j}=\left\{\begin{array}{ll} 
\pm 2, & j=\left(j_{m^{\prime}}+j_{m^{\prime}+1}\right) / 2 \\
0, & \text { otherwise }
\end{array}, \quad k_{m}=\left\{\begin{array}{ll}
\mp 1, & m=m^{\prime} \\
\mp 1, & m=m^{\prime}+1 \\
0, & \text { otherwise }
\end{array} ;\right.\right.
$$

(3) $l_{j}=0$ for $j \in \mathbb{N}_{*}^{1} \cup \mathbb{N}_{*}^{2}$, and

$$
k_{m}=\left\{\begin{array}{ll}
0, & 1 \leq m<n \\
-\sum_{j \in \mathbb{N}_{*}} l_{j}, & m=n
\end{array} .\right.
$$

It's easy to check that the first inequality of (8.51) holds true for all of the above three cases. Therefore, the first part of the assumption $\left(\mathrm{D}^{*}\right)$ holds true.

Now we consider sup norm and Lipschitz semi-norm of the perturbation

$$
P=Q+R+\varepsilon K
$$

on $D(s, r) \times \Pi$, where $D(s, r)$ is defined in (1.2) by letting $p=N+1 / 2$ and $\Pi$ is defined in (8.42). We choose $s>0$ a constant, and

$$
r=\varepsilon^{1 / 4} .
$$

In view of (8.41), we have

$$
\left\|X_{Q}\right\|_{r, a, p-1, D(s, r) \times \Pi}=O\left(r^{2}\right)=O\left(\varepsilon^{1 / 2}\right) .
$$


In view of (8.12), we know $R$ is at least five order of $q$. Thus,

$$
\left\|X_{R}\right\|_{r, a, p-1, D(s, r) \times \Pi}=O\left(\left(\varepsilon^{1 / 5}\right)^{5} r^{-2}\right)=O\left(\varepsilon^{1 / 2}\right) .
$$

In view of (8.34), we have

$$
\left\|X_{K}\right\|_{r, a, p-1, D(s, r) \times \Pi}=O\left(r^{-2}\right)=O\left(\varepsilon^{-1 / 2}\right) .
$$

From (8.55) (8.56) (8.57) we get

$$
\left\|X_{P}\right\|_{r, a, p-1, D(s, r) \times \Pi}=O\left(\varepsilon^{1 / 2}\right) .
$$

Since $X_{P}$ is real analytic in $\xi$, we have

$$
\left\|X_{P}\right\|_{r, a, p-1, D(s, r) \times \Pi}^{\text {lip }}=O\left(\varepsilon^{1 / 2} \varepsilon^{-2 / 5}\right) .
$$

We choose

$$
\alpha=\varepsilon^{9 / 20} \gamma^{-1}, \quad \beta=1 / 18
$$

where $\gamma$ is taken from the KAM theorem. Set $M:=M_{1}+M_{2}$, which only depends on the set $J$. It's obvious that when $\varepsilon$ is small enough,

$$
\tilde{\varepsilon}:=\left\|X_{P}\right\|_{r, a, p-1, D(s, r) \times \Pi}+\frac{\alpha}{M}\left\|X_{P}\right\|_{r, a, p-1, D(s, r) \times \Pi}^{\text {lip }}=O\left(\varepsilon^{1 / 2}\right) \leq(\alpha \gamma)^{1+\beta},
$$

which is just the smallness condition (1.7). Now the conclusion of Theorem 8.2 follows from Theorem 1.2.

\section{A Technical Lemma}

Lemma 9.1. Let $F=\left(F_{i j}\right)_{i, j \geq 1}$ be a bounded operator on $\ell^{2}$ which depends on $x \in \mathbb{T}^{n}$ such that all elements $\left(F_{i j}\right)$ are analytic on $D(s)$. Suppose $R=\left(R_{i j}\right)_{i, j \geq 1}$ is another operator on $\ell^{2}$ depending on $x$ whose elements satisfy

$$
\sup _{x \in D\left(s^{\prime}\right)}\left|R_{i j}(x)\right| \leq \frac{1}{|i-j|} \sup _{x \in D(s-\sigma)}\left|F_{i j}(x)\right|, \quad i \neq j,
$$

and $R_{i i}=0$. Then $R$ is a bounded operator on $\ell^{2}$ for every $x \in D\left(s^{\prime}\right)$, and

$$
\sup _{x \in D\left(s^{\prime}\right)}\|R(x)\| \leq \frac{4^{n+1}}{\sigma^{n}} \sup _{x \in D(s)}\|F(x)\| .
$$

Proof. This is lemma M.3 in [10].

Acknowledgement. The authors are very grateful to the referees for their invaluable suggestions.

\section{References}

[1] Ablowitz, M. J., Fokas, A. S.: The inverse scattering transform for the Benjamin-Ono equation, a pivot for multidimensional problems, Stud. Appl. Math. 68, 1-10 (1983)

[2] Bambusi, D.; Graffi, S. Time Quasi-Periodic Unbounded Perturbations of Schrödinger Operators and KAM Methods. Commun. Math. Phys. 219, 465-480 (2001) 
[3] Benjamin, T. B.: Internal waves of permanent form in fluids of great depth. J. Fluid Mech. 29, 559-592 (1967)

[4] Bourgain, J.: Recent progress on quasi-periodic lattice Schrödinger operators and Hamiltonian PDEs. Russian Math. Surveys 59(2), 231-246 (2004)

[5] Klainerman, S.: Long-time behaviour of solutions to nonlinear wave equations. In Proceedings of International Congress of Mathematics (Warsaw), 1209-1215 (1983)

[6] Kuksin, S. B.: On small-denominators equations with large varible coefficients. J. Appl. Math. Phys.(ZAMP) 48, 262-271 (1997)

[7] Kuksin, S. B.: Analysis of Hamiltonian PDEs, Oxford Univ. Press, Oxford, 2000

[8] Kuksin, S. B.: Fifteen years of KAM in PDE, "Geometry, topology, and mathematical physics": S. P. Novikov's seminar 2002-2003 edited by V. M. Buchstaber, I. M. Krichever. Amer. Math. Soc. Transl. Series 2, Vol.212, 2004, pp.237-257

[9] Kuksin, S. B., Pöschel, J: Invariant Cantor manifolds of quasi-periodic oscillations for a nonlinear Schrödinger equation. Ann. Math. 143, 147-179 (1996)

[10] Kappeler, T., Pöschel, J.: KdV\&KAM. Springer-Verlag, Berlin Heidelberg, 2003

[11] Lax, P. D.: Development of singularities of solutions of nonlinear hyperbolic partial differential equations. J. Math. Phys. 5, 611-613 (1964)

[12] Liu, J., Yuan, X.: Spectrum for quantum Duffing oscillator and small-divisor equation with large variable coefficient. Commun. Pure. Appl. Math. 63, 1145-1172 (2010)

[13] Molinet, L.: Global well-posedness in the energy space for the Benjamin-Ono equation on the circle. Math. Ann. 337, 353-383 (2007)

[14] Molinet, L.: Global well-posedness in $L^{2}$ for the periodic Benjamin-Ono equation. Amer. J. Math. 130, 635-683 (2008) 